\title{
Precision Medicine in Oncology: A Review of Multi-Tumor Actionable Molecular Targets with an Emphasis on Non-Small Cell Lung Cancer
}

\author{
Matthew K. Stein ${ }^{1}$, Oluchukwu Oluoha ${ }^{2}$, Kruti Patel ${ }^{2}$ and Ari VanderWalde ${ }^{3, *}$ \\ 1 Missouri Baptist Medical Center, Heartland Cancer Research, NCI Community Oncology Research Program, \\ St. Louis, MO 63131, USA; Matthew.stein@bjc.org \\ 2 Division of Hematology and Oncology, University of Tennessee Health Science Center, Memphis, TN 38103, \\ USA; ooluoha@westclinic.com (O.O.); kpatel@westclinic.com (K.P.) \\ 3 West Cancer Center and Research Institute, Germantown, TN 38138, USA \\ * Correspondence: avanderwalde@westclinic.com
}

Citation: Stein, M.K.; Oluoha, O.; Patel, K.; VanderWalde, A. Precision Medicine in Oncology: A Review of Multi-Tumor Actionable Molecular Targets with an Emphasis on Non-Small Cell Lung Cancer. J. Pers. Med. 2021, 11, 518. https://doi.org/ 10.3390/jpm11060518

Academic Editor: Luigi Minafra

Received: 29 April 2021

Accepted: 2 June 2021

Published: 5 June 2021

Publisher's Note: MDPI stays neutral with regard to jurisdictional claims in published maps and institutional affiliations.

Copyright: (c) 2021 by the authors. Licensee MDPI, Basel, Switzerland. This article is an open access article distributed under the terms and conditions of the Creative Commons Attribution (CC BY) license (https:// creativecommons.org/licenses/by/ $4.0 /)$.

\begin{abstract}
Precision medicine is essential for the modern care of a patient with cancer. Comprehensive molecular profiling of the tumor itself is necessary to determine the presence or absence of certain targetable abnormalities or biomarkers. In particular, lung cancer is a disease for which targetable genomic alterations will soon guide therapy in the majority of cases. In this comprehensive review of solid tumor-based biomarkers, we describe the genomic alterations for which targeted agents have been approved by the United States Food and Drug Administration (FDA). While focusing on alterations leading to approvals in a tumor-agnostic fashion (MSI-h, TMB-h, NTRK) and on those alterations with approvals in multiple malignancies (BRAF, ERBB2, RET, BRCA, PD-L1), we also describe several biomarkers or indications that are likely to lead to an approved drug in the near future (e.g., KRAS G12C, PD-L1 amplification, HER2 overexpression in colon cancer, HER2 mutations in lung cancer). Finally, we detail the current landscape of additional actionable alterations (EGFR, $A L K, R O S 1, M E T$ ) in lung cancer, a biomarker-rich malignancy that has greatly benefitted from the precision oncology revolution.
\end{abstract}

Keywords: cancer; next-generation sequencing; targeted therapy; precision oncology; tumor-agnostic indications; solid tumors; tumor markers; FDA-approved therapeutics

\section{Introduction}

Precision medicine, defined as supplying the right treatment to the right patient at the right time, has become an essential element of cancer care, Taking advantage of novel technologies developed following sequencing of the human genome approximately twenty years ago, precision oncology leverages a tumor's molecular features with available and novel therapeutics [1-4]. Prior to the advancement of comprehensive tumor profiling, successful implementation of a precision oncology approach included tyrosine kinase inhibitors (TKIs) imatinib for breakpoint cluster region-Abelson ( $B C R-A B L)$-rearranged chronic myeloid leukemia [5] and trastuzumab for human epidermal growth factor 2 (HER2) immunohistochemistry (IHC) overexpressed or amplified breast cancer [6,7].

Currently, molecular profiling is available to comprehensively characterize a patient's tumor within as few as two weeks and includes interrogation of anywhere from hundreds of genes to the whole exome for mutations, insertions, deletions or copy-number alterations via next-generation sequencing (NGS), gene fusions with RNA sequencing and various protein changes with IHC. The goal of such extensive testing is to unveil the genomic makeup of a patient's tumor, which can inform the most effective therapeutic approach. Oftentimes, this must be coupled with the malignancy's site-of-origin and histologic features; however, precision treatment strategies are increasingly being employed in a 
tissue-agnostic fashion through a growing list of pan-tumor United States Food and Drug Administration (FDA) approvals, clinical trials and off-label use when a molecular approach can be justified (e.g., through a tumor board consensus) [8].

In this review, we will outline progress in precision medicine in oncology, with an aim to summarize the current landscape of FDA-approved therapies based upon predictive molecular biomarkers in solid tumors, focusing on those markers that determine therapeutic options in more than one malignancy type. As will be seen, several pan-tumor and multi-tumor FDA approvals exist for both tumors with alterations expressing sensitivity to targeted agents (including TKIs and antibody-drug conjugates (ADCs)) as well as immune checkpoint inhibitors (ICIs). The full extent of molecular targets in cancer cannot be completely summarized in a single paper, and we will concentrate on those findings deemed DNA-based alterations by most NGS vendors.

We will conclude by switching from biomarker to malignancy, detailing present treatment options available for non-small cell lung cancer (NSCLC). NSCLC serves as a model for precision oncology where patients can benefit substantially from employment of molecular profiling.

\section{Molecular Alterations with Approvals Regardless of Tumor-Site}

To date, three molecular targets (microsatellite instability-high (MSI-H) or mismatch repair deficient (MSI-H/dMMR), neurotrophic tropomyosin-related kinase (NTRK1/2/3) fusions or high tumor mutational burden (TMB-H)), have led to four site- or tumor-agnostic approvals by the FDA. These biomarkers and drugs with corresponding FDA approvals are listed in the first part of Table 1.

Table 1. Molecular alterations guiding therapy with agents approved in multiple tumor types.

\begin{tabular}{|c|c|c|c|c|c|}
\hline Alteration(s) & Indication & Line of Therapy & Medications & Drug Class & FDA Approval Date \\
\hline \multirow{5}{*}{$\begin{array}{l}\text { MSI-h/ } \\
\text { dMMR }\end{array}$} & Any tumor type & $\begin{array}{l}>\text { 2nd line } \\
\text { metastatic }\end{array}$ & Pembrolizumab & PD-1 mAb & 23 May 2017 [9] \\
\hline & \multirow{3}{*}{ Colorectal cancer } & 2nd line metastatic & Nivolumab & PD-1 mAb & 31 July 2017 [10] \\
\hline & & 2nd line metastatic & $\begin{array}{l}\text { Ipilimumab + } \\
\text { Nivolumab }\end{array}$ & $\begin{array}{l}\text { CTLA-4 mAb + } \\
\text { PD-1 mAb }\end{array}$ & 10 July 2018 [11] \\
\hline & & 1st line metastatic & Pembrolizumab & PD-1 mAb & 29 June 2020 [12] \\
\hline & $\begin{array}{l}\text { Endometrial } \\
\text { cancer }\end{array}$ & $\begin{array}{l}\text { Progression after } \\
\text { platinum }\end{array}$ & Dostarlimab & PD-1 mAb & 22 April 2021 [13] \\
\hline $\begin{array}{c}\text { TMB-h } \\
(>10 \mathrm{mut} / \mathrm{Mb})\end{array}$ & Any tumor type & $\begin{array}{l}\text { >2nd line } \\
\text { metastatic }\end{array}$ & Pembrolizumab & PD-1 mAb & 16 June 2020 [14] \\
\hline \multirow{2}{*}{ NTRK fusions } & \multirow{2}{*}{ Any tumor type } & \multirow{2}{*}{ Any line } & Larotrectinib & Trk inhibitor & 26 November 2018 [15] \\
\hline & & & Entrectinib & Trk inhibitor & 15 August 2019 [16] \\
\hline \multirow{7}{*}{ BRAF V600 mt } & \multirow{7}{*}{ Melanoma } & \multirow{6}{*}{ Any metastatic line } & Vemurafenib $^{1}$ & BRAF inhibitor & 17 August 2011 [17] \\
\hline & & & Dabrafenib $^{1}$ & BRAF inhibitor & 29 May 2013 [18] \\
\hline & & & Trametinib $^{1}$ & MEK inhibitor & 29 May 2013 [18] \\
\hline & & & $\begin{array}{l}\text { Vemurafenib + } \\
\text { Cobimetinib }\end{array}$ & BRAF + MEK & 10 November 2015 [19] \\
\hline & & & $\begin{array}{c}\text { Dabrafenib + } \\
\text { Trametinib }\end{array}$ & BRAF + MEK & 9 January 2014 [20] \\
\hline & & & $\begin{array}{l}\text { Encorafenib + } \\
\text { Binimetinib }\end{array}$ & BRAF + MEK & 27 June 2018 [21] \\
\hline & & Adjuvant & $\begin{array}{c}\text { Dabrafenib + } \\
\text { Trametinib }\end{array}$ & BRAF + MEK & 30 April 2018 [22] \\
\hline
\end{tabular}


Table 1. Cont.

\begin{tabular}{|c|c|c|c|c|c|}
\hline Alteration(s) & Indication & Line of Therapy & Medications & Drug Class & FDA Approval Date \\
\hline & & 1st line & $\begin{array}{l}\text { Atezolizumab, } \\
\text { Vemurafenib and } \\
\text { Cobimetinib }\end{array}$ & $\begin{array}{c}\text { PD-L1 + BRAF + } \\
\text { MEK }\end{array}$ & 30 July 2020 [23] \\
\hline & NSCLC & 2nd line & $\begin{array}{c}\text { Dabrafenib + } \\
\text { Trametinib }\end{array}$ & BRAF + MEK & 22 June 2017 [24] \\
\hline & Anaplastic thyroid & Any line & $\begin{array}{l}\text { Dabrafenib + } \\
\text { trametinib }\end{array}$ & BRAF + MEK & 4 May 2018 [25] \\
\hline & Colorectal & 2nd line & $\begin{array}{c}\text { Encorafenib + } \\
\text { cetuximab }\end{array}$ & $\begin{array}{l}\text { BRAF inh + } \\
\text { EGFR } \mathrm{mAb}\end{array}$ & 8 April 2020 [26] \\
\hline \multirow{9}{*}{$\begin{array}{l}\text { HER2 (ERBB2) } \\
\text { overexpression }\end{array}$} & \multirow{7}{*}{ Breast $^{2}$} & \multirow{2}{*}{$\begin{array}{l}\text { Neoadjuvant, } \\
\text { adjuvant or } \\
\text { metastatic }\end{array}$} & Trastuzumab & Anti HER2 mAb & 25 September 1998 [27] \\
\hline & & & Pertuzumab & Anti HER2 mAb & 8 June 2012 [28] \\
\hline & & \multirow[t]{2}{*}{$\begin{array}{l}\text { Adjuvant or } \\
\text { metastatic }\end{array}$} & $\begin{array}{l}\text { Ado-trastuzumab } \\
\text { emtansine } \\
\text { (TDM-1) }\end{array}$ & $\begin{array}{l}\text { Antibody drug } \\
\text { conjugate }\end{array}$ & 22 February 2013 [29] \\
\hline & & & Neratinib & Small molecule & 17 July 2017 [30] \\
\hline & & \multirow{3}{*}{ Metastatic } & $\begin{array}{c}\text { Fam-trastuzumab } \\
\text { deruxtecan }\end{array}$ & $\begin{array}{c}\text { Antibody drug } \\
\text { conjugate }\end{array}$ & 20 December 2019 [31] \\
\hline & & & Lapatinib & Small molecule & 13 March 2007 [32] \\
\hline & & & Tucatinib & Small molecule & 17 April 2020 [33] \\
\hline & & & Trastuzumab & Anti HER2 mAb & 20 October 2010 [34] \\
\hline & Gastric/GEJ & 1st line metastatic & $\begin{array}{c}\text { Fam-trastuzumab } \\
\text { deruxtecan }\end{array}$ & $\begin{array}{c}\text { Antibody drug } \\
\text { conjugate }\end{array}$ & 15 Januray 2021 [35] \\
\hline \multirow{4}{*}{ RET alterations } & $\begin{array}{c}\text { Medullary thyroid } \\
\text { cancer } \\
\text { (RET-mutated) }\end{array}$ & Metastatic & Selpercatinib & \multirow{4}{*}{$\begin{array}{l}\text { Small molecule } \\
\text { RET inhibitor }\end{array}$} & 8 May 2020 [36] \\
\hline & $\begin{array}{c}\text { Any thyroid cancer } \\
\text { (RET fusion) }\end{array}$ & Metastatic & Selpercatinib & & 8 May 2020 [36] \\
\hline & \multirow{2}{*}{$\begin{array}{l}\text { NSCLC (RET } \\
\text { fusion) }\end{array}$} & \multirow{2}{*}{ Metastatic } & Selpercatinib & & 8 May 2020 [36] \\
\hline & & & Pralsetinib & & 4 September 2020 [37] \\
\hline \multirow{5}{*}{$\begin{array}{c}\text { DNA repair } \\
\text { deficiency } \\
\text { (Either BRCA1/2 } \\
\text { somatic mutations, } \\
\text { BRCA1/2 germline } \\
\text { mutations, } \\
\text { homologous repair } \\
\text { deficiency (HRD) } \\
\text { or homologous } \\
\text { recombination } \\
\text { repair mutations } \\
\text { (HRR) }\end{array}$} & \multirow{3}{*}{ Ovarian cancer } & \multirow{3}{*}{$\begin{array}{l}\text { 1st line or late-line } \\
\text { maintenance }\end{array}$} & $\begin{array}{c}\text { Olaparib } \\
\text { (BRCA germline, } \\
\text { somatic or HRD) }\end{array}$ & PARP inhibitor & $\begin{array}{c}19 \text { December } 2018 \\
\text { (BRCA), } 8 \text { May } 2020 \\
\text { (HRD) [38] }\end{array}$ \\
\hline & & & $\begin{array}{c}\text { Rucaparib (initially } \\
\text { for BRCA } \\
\text { mutation, now } \\
\text { regardless of } \\
\text { biomarker status) }\end{array}$ & PARP inhibitor & $\begin{array}{l}19 \text { December } 2016 \\
\text { (BRCA) [39] } 6 \text { April } \\
2018 \text { (regardless of } \\
\text { biomarker) [40] }\end{array}$ \\
\hline & & & $\begin{array}{c}\text { Niraparib } \\
\text { (regardless of } \\
\text { biomarker status } \\
\text { for maintenance, } \\
\text { late line for HRD) }\end{array}$ & PARP inhibitor & $\begin{array}{c}27 \text { March } 2017 \\
\text { (regardless of } \\
\text { biomarker) [41] } \\
\text { 10/23/2019 (HRD) } \\
{[42]}\end{array}$ \\
\hline & \multirow{2}{*}{ Breast cancer } & \multirow{2}{*}{$\begin{array}{l}>2 \text { nd line } \\
\text { metastatic }\end{array}$} & $\begin{array}{c}\text { Olaparib } \\
\text { (BRCA germline } \\
\text { only) }\end{array}$ & PARP inhibitor & 12 January 2018 [43] \\
\hline & & & $\begin{array}{l}\text { Talazoparib (BRCA } \\
\text { germline only) }\end{array}$ & PARP inhibitor & 16 October 2018 [44] \\
\hline
\end{tabular}


Table 1. Cont.

\begin{tabular}{cccccc}
\hline Alteration(s) & Indication & Line of Therapy & Medications & Drug Class & FDA Approval Date \\
\hline Pancreatic cancer & $\begin{array}{c}\text { Metastatic } \\
\text { maintenance }\end{array}$ & $\begin{array}{c}\text { Olaparib } \\
\text { (BRCA germline } \\
\text { only) }\end{array}$ & PARP inhibitor & 30 December 2019 [45] \\
\cline { 2 - 6 } & Metastatic & $\begin{array}{c}\text { Rucaparib } \\
\text { (BRCA germline or } \\
\text { somatic) }\end{array}$ & PARP inhibitor & 15 May 2020 [46] \\
\cline { 3 - 6 } & & $\begin{array}{c}\text { Olaparib } \\
\text { (HRR germline or } \\
\text { somatic) }\end{array}$ & PARP inhibitor & 19 May 2020 [47] \\
\hline
\end{tabular}

${ }^{1}$ No longer preferred as single agents in this disease. ${ }^{2}$ Many HER2 agents may be used in combination with other HER2 agents or with chemotherapy, depending on the indication. Approvals given for HER2 agents are for the first approval of each drug.

\section{1. $M S I-h / d M M R$}

The first of these approvals occurred on 23 May 2017 when pembrolizumab, an ICI whose activity lies in the inhibition of programmed cell death protein 1 (PD-1), was approved for second-line or later treatment of metastatic or unresectable solid tumors in pediatric and adult patients found to be MSI-H/dMMR [9]. The basis of the approval was from 149 MSI-H/dMMR patients spanning five uncontrolled, multi-cohort, multicenter, single-arm clinical trials (KEYNOTE-012, -016, -028, -158 and -164) [9,48-51], where the overall response rate (ORR) was $40 \%$ (95\% confidence interval (CI): $31.7,47.9)$ and responses lasted for $>6$ months in $78 \%$ of responders. In this cohort, $90 / 149(60 \%)$ of patients had colorectal cancer, for which prior treatment with a fluoropyrimidine, oxaliplatin and irinotecan was required; 14 other tumor types were evaluated leading up to the 2017 FDA approval.

Several other ICI agents are FDA approved for MSI-H/dMMR colorectal cancer patients. These include nivolumab following treatment with a fluoropyrimidine, oxaliplatin and irinotecan (July 2017; CheckMate 142) [52]; nivolumab and ipilimumab after a fluoropyrimidine, oxaliplatin and irinotecan (July 2018; CheckMate 142) [53]; and pembrolizumab as the first frontline approval in MSI-H/dMMR colorectal cancer (June 2020; KEYNOTE177) [54]. While the tumor site-agnostic approval was groundbreaking, recently published data from KEYNOTE-158 showed a wide range of response rates to pembrolizumab in this setting based on primary tumor site [51], with the highest responses seen in endometrial cancer $(57 \%)$ and the lowest with pancreatic $(18 \%)$ and central nervous system $(0 \%)$ malignancies. While larger cohorts and further study is needed into the molecular mechanisms of response [55], these findings suggest the site-agnostic model of ICI use should be evaluated thoughtfully in these and other MSI-H/dMMR solid tumors when other treatment options exist [56].

\subsection{NTRK1/2/3 Fusions}

NRTK fusions occur in $<0.5 \%$ of all cancer types, but are enhanced in some rare cancers or those with atypical histology, including salivary carcinoma (5\%), thyroid (2\%), sarcoma (including uterine, $1 \%)$ and possibly glioblastoma multiforme $(<1 \%)$ [57]. The FDA approved larotrectinib [15] on 26 November 2018 and entrectinib [16] on 15 August 2019 for adult and pediatric solid tumor patients whose metastatic or unresectable tumors do not contain a resistance mutation to either agent at the start of treatment. Approval of larotrectinib was based upon the efficacy observed in the first 55 patients of three multicenter, open-label, single-arm clinical trials (LOXO-TRK-14001, SCOUT, NAVIGATE), where Drilon et al. reported a response rate of $75 \%$, which was ongoing in $71 \%$ of responders at one year [58]. Comprising a cohort of 17 different tumor types, patients harboring a TRK fusion and treated with larotrectinib primarily had grade 1 toxicities including increased liver enzymes, fatigue, nausea, vomiting and anemia. In a recent pooled analysis of a larger cohort from the same three trials, an ORR was found to be $79 \%$ for 153 evaluable 
patients, with $16 \%$ complete responses and a median duration of response of over 35 months [59]. Additionally, long-term toxicity data showed the safety of larotrectinib with no treatment-related deaths observed and the most frequent grade 3 or 4 toxicities were increased alanine aminotransferase levels (3\%), neutropenia (2\%) and anemia $(2 \%)$.

The efficacy of entrectinib for patients with advanced solid tumors with NTRK fusions was likewise recently described in a combined analysis from three ongoing phase 1 and 2 clinical trials (ALKA-372-001, STARTRK-1 and STARTRK-2). Doebele and colleagues described a $57 \%$ response rate with a median duration of 10 months in a cohort of 54 patients ( $>60$ had received prior systemic therapy) comprising 19 different histologies [60]. It should also be noted that in addition to activity against NTRK fusions, entrectinib is also FDA approved for the treatment of ROS proto-oncogene 1 (ROS1) rearranged NSCLC.

The robust efficacy of larotrectinib and entrectinib with a tolerable safety profile makes both agents attractive for NTRK fusion-positive metastatic cancer patients. Upon progression, molecular profiling should be repeated to assess for resistance mechanisms, as NTRK kinase domain mutations (involving solvent front, gatekeeper residue or xDFG motif) or off-target mutations (including Kirsten rat sarcoma viral oncogene homolog (KRAS) mutations, mesenchymal-epithelial transition (MET) amplifications and serine-threonine protein kinase B-RAF (BRAF) V600E mutations) can inform the next systemic approach [61]. If progression is restricted to a limited number of sites (termed oligometastatic), local therapy with continued NTRK inhibition can be pursued. However, if extensive progression occurs and NTRK resistance mutations are identified, next-generation TKIs repotrectinib $[62,63]$ and seltrectinib [64] are currently being evaluated and show preliminary efficacy in this setting.

\subsection{Tumor Mutational Burden}

On 16 June 2020, the FDA approved pembrolizumab for its second tumor site-agnostic indication. Specifically, pembrolizumab can be considered following progression on another treatment for all metastatic or unresectable adult and pediatric solid tumor patients found to have high tumor mutational burden (TMB-H), defined as $\geq 10$ mutations/megabase (mut/Mb). As a part of this approval, the FoundationOne CDx assay (Foundation Medicine, Cambridge, MA, USA) was authorized as a companion diagnostic for tissue TMB evaluation [14]. The approval was based upon recently published prospective data from 10 treatment-refractory cohorts in the phase 2 KEYNOTE-158 trial, which showed an ORR of $29 \%$ of $102 \mathrm{TMB}-\mathrm{H}$ patients to pembrolizumab, compared to $6 \%$ of those with $\mathrm{TMB}<10 \mathrm{mut} / \mathrm{Mb}$ [65].

The pan-tumor approval for TMB-H has been controversial in the oncology community. On one hand, some point to potential flaws in the study including a perceived arbitrary $\mathrm{TMB}$ cutoff of $10 \mathrm{mut} / \mathrm{Mb}$, the lack of a control arm in KEYNOTE-158 and the composition of tumor types included in the $102 \mathrm{TMB}-\mathrm{H}$ patients (i.e., $>60 \%$ were small cell lung, endometrial and cervical, which already have ICIs approved in some capacity; less commonly enrolled tumor types need a higher sample size to determine efficacy) [66]. To this end, McGrail and colleagues recently published retrospective data from The Cancer Genome Atlas (TCGA) which suggests a blanket TMB cutoff of 10 mut/Mb for pembrolizumab us may not be applicable in all solid tumor types. The authors first divided solid tumor patients into two categories, those with a positive versus no correlation between CD8 T-cell levels and neoantigen load, a term referring to the presumption that higher amounts of tumor mutations lead to certain antigen peptides which in turn activate the immune system. While TMB-H predicted a response to ICI in those with a correlation between CD8 T-cells and neoadjuvant load (e.g., melanoma, bladder and lung cancer), this benefit was not seen in those tumor types where no such correlation was seen (e.g., breast, prostate, glioma). In fact, the ORR for TMB-H patients in this latter subgroup was $<20 \%$, and TMB-H patients were actually found to have a lower ORR than those with low TMB (odds ratio $(\mathrm{OR})=0.46,95 \%$ CI $0.24-0.88, p=0.02)$ [67]. 
In contrast, advocates of utilizing TMB-H such as Subbiah and colleagues cite the durability of responses seen in KEYNOTE-158 (approximately half were for at least two years), the resultant expansion of genomic profiling to include rare tumors, improvement of pembrolizumab reimbursement and access given FDA approval, access for minority and underserved populations and overall enabling of patients and physicians to make informed clinical decisions [68]. As the careful use of TMB-H makes its way to the clinic, emerging co-occurring biomarkers, such as mutations in DNA polymerase epsilon (POLE) and delta 1 (POLD1) may help predict survival with ICI use [69], though no FDA approvals have yet been based on these alterations.

\subsection{On the Horizon: PD-L1 Amplification}

While requiring further study, additional biomarkers obtained from comprehensive molecular profiling may eventually be considered for predictive pan-tumor use. One such biomarker includes amplification of PD-L1 (or CD274), which Goodman et al. identified in $0.7 \%$ of 118,000 profiled solid tumor patients and may predict efficacy to ICI [70]. This is in contrast to PD-L1 expression measured using immunohistochemistry, which is both more common and less predictive (see PD-L1 section below). With only limited published case reports or series to date [71-73], there does appear to be a histologic-dependent range in frequency of $P D-L 1$ amplifications with increased incidence in uncommon tumor types including bladder squamous cell, renal sarcomatoid, liver mixed hepatocellular and anaplastic thyroid carcinoma (all $>5 \%$ ) [70].

\section{Molecular Alterations with Approvals in Multiple Tumor Types}

While not approved in a site-agnostic fashion, a growing compendium of molecular biomarkers are susceptible to precision oncology therapies with FDA approval in more than one tumor type. The latter part of Table 1 provides a summary of the agents which are discussed below.

\subsection{BRAF V600 Mutations}

\subsubsection{Melanoma}

Mutations in the 600th amino acid position of $B R A F$ activate the mitogen-activated protein (MAP) kinase pathway, leading to cancer cell growth and proliferation. These lesions, predominantly V600E or V600K, can be identified in multiple solid tumors using NGS or other sequencing panels and are targetable. The prototype for targeting $B R A F$ V600 lesions is cutaneous melanoma, where BRAF mutations occur in $40-60 \%$ and the single-agent selective BRAF-inhibitor, vemurafenib, was approved a decade ago [74]. Shortly following the FDA approval of vemurafenib, the single-agent BRAF-inhibitor dabrafenib [75] and the single-agent mitogen-activated protein kinase (MEK)-inhibitor trametinib [76] likewise showed activity, including activity against brain metastases in the case of dabrafenib [77], and both received approval in 2013. Due to the superior efficacy of alternative combination regimens and ICIs (see below), single-agent BRAF inhibition is currently given only if other agents are contraindicated while trametinib monotherapy is no longer recommended in BRAF mutant melanoma.

Predictable resistance to single-agent $B R A F$ V600 inhibitors occurs through various means of reactivation of the MAP kinase pathway including paradoxical activation of downstream MEK [78]. Therefore, dual pathway blockade with both a BRAF and a MEK inhibitor has since become the predominant targeted approach to BRAF-mutant melanoma. First-line phase 3 trials comparing BRAF/MEK combinations to single-agent targeted therapy have shown an overall survival (OS) advantage for dual therapy and have led to the FDA approvals of dabrafenib and trametinib in 2014 (COMBI-d, COMBI-v) [79-83], vemurafenib and cobimetinib in 2015 (CO-BRIM) [84,85] and encorafenib and binimetinib in 2018 (COLUMBUS) [86-88]. For example, long-term follow-up of encorafenib and binimetinib showed a 34-month median OS with this combination versus 17 months with vemurafenib, amounting to a 39\% decreased risk of death (HR 0.61; 95\% CI, 0.48-0.79) [88]. 
While all three combinations are FDA approved and have ORRs of approximately $60-70 \%$ (vs. 50\% for single-agent), median PFS of 11-15 months (vs. 7-10 months), the combination of encorafenib and binimetinib may be more tolerable with reduced pyrexia, fatigue and other symptoms [89].

It should also be noted that dabrafenib and trametinib were FDA approved in 2018 for the adjuvant treatment of $B R A F \mathrm{~V} 600 \mathrm{E}$ or $\mathrm{V} 600 \mathrm{~K}$ mutations following resection, which was based upon the COMBI-AD phase 3 study [90]. This represents one of the first approvals of a targeted agent for early-stage disease following surgery (with the exception of HER2 therapy for breast cancer; see below). Recently, Dummer et al. showed 5-year followup data, noting a $49 \%$ reduction for relapse or death for patients treated with planned 12 months of adjuvant dabrafenib and trametinib versus placebo [91].

Advances in targeted therapy for BRAF-mutated melanoma occurred at the same time as the development of ICI. In light of this, several additional points should be made in the management of BRAF-mutant melanoma. First, while BRAF V600 mutations predict response to targeted therapy, patients are also eligible to receive ICI in either the adjuvant [92,93] or metastatic [94-98] setting regardless of BRAF mutation status. Additionally, the IMspire150 trial was recently published, leading to the approval of the combination of agent atezolizumab (anti-PD-L1) and the BRAF/MEK inhibitors vemurafenib and cobimetinib in first-line metastatic disease [99]. The decision of whether to treat metastatic patients with ICIs, anti-BRAF/MEK agents or a combination of the two is not standardized, but involves shared-decision making with the patient and considerations such as toxicity differences, disease aggressiveness and pace, metastatic distribution including brain metastases, lactate dehydrogenase level and other clinical factors. Finally, it should be noted that only appropriate BRAF mutations (namely V600, with rare exceptions) are selected for targeted therapy, as utilization of BRAF/MEK inhibitors for certain non-V600 (e.g., K601E) lesions can paradoxically activate the MAP kinase pathway and possibly result in poor outcomes [100-102].

\subsubsection{Lung Cancer}

In June 2017, the FDA approved the combination of dabrafenib and trametinib for metastatic NSCLC patients harboring BRAF V600E mutations based upon the international, multicenter, three-cohort, non-randomized, open-label BRF113928 trial [103-105]. Overall, 93 metastatic NSCLC patients were treated, with 36 receiving no prior therapy. Previously untreated patients showed an ORR of $64 \%$ with the majority partial responses; $69 \%$ had at least one grade 3-4 adverse event, including pyrexia, hypertension, increase in alanine aminotransferase and vomiting [105]. Subsequent molecular profiling of new tissue or liquid samples after progression has been described to show resistance mechanisms in MAP kinase signaling, such as MEK, KRAS and NRAS mutations [106].

\subsubsection{Thyroid Cancer}

$B R A F$ V600E mutations are frequent in differentiated thyroid cancer, occurring in almost $50 \%$ of papillary disease and associated with poor prognosis, especially when co-occurring with TERT promoter mutations $[107,108]$. For metastatic differentiated thyroid cancer patients harboring BRAF V600E mutations who are refractory to radioactive iodine, targeted therapy with BRAF-inhibitors dabrafenib or vemurafenib can be considered $[109,110]$. Anaplastic thyroid carcinoma is typically highly aggressive with a 1-year survival of roughly $20 \%$ [111]. Harboring BRAF V600 mutations in roughly 20-50\% [112], Subbiah et al. showed a promising ORR of $69 \%$ (11 of 16) with dabrafenib and trametinib [113], ultimately leading to FDA approval of this BRAF/MEK combination for unresectable anaplastic thyroid carcinoma in 2018.

\subsubsection{Colorectal Cancer}

Finally, on 8 April 2020, the FDA approved the doublet encorafenib and cetuximab for metastatic colorectal cancer containing a BRAF V600E mutation after receipt of prior 
therapy [114]. The approval was based on data from the randomized, phase 3 BEACON CRC trial, where both the triplet (encorafenib, cetuximab and MEK inhibitor binimetinib) and doublet (encorafenib plus cetuximab) arms similarly improved ORR, PFS and OS versus standard-of-care cetuximab plus irinotecan-based regimens. Updated analysis showed a similar median PFS of 4.5 months for the triplet arm and 4.3 months for the doublet arm, both superior to 1.5 months for control [115]. With similar efficacy and reduced toxicity including gastrointestinal and hematologic compared to the triplet, preference for FDA approval was given to doublet encorafenib and cetuximab in advanced BRAF V600E colorectal cancer.

\subsection{ERBB2/HER2}

\subsubsection{Breast and Gastric Cancer}

Up to $20 \%$ of breast [116] and 13\% of gastric cancers [117] overexpress the HER2 protein. That HER2 expression is a negative prognostic factor in breast and gastric cancer has been long recognized [118,119]. Generally, HER2 overexpression is determined by IHC testing (defined as $3+$ ) and confirmed by reflex in-situ hybridization (ISH) testing for tumors with equivocal (2+) IHC results. Historically, tumors with 0 or $1+$ expression by IHC have been denoted HER2-negative. Since 1998, trastuzumab, a monoclonal antibody targeting the HER2 protein has been available for the treatment of metastatic disease based on a number of studies [7,120-122]. Afterward, HER2 therapy with trastuzumab expanded into the adjuvant and neoadjuvant settings [123-125].

Numerous other HER-2 directed agents are now available. For example, in the metastatic setting, lapatinib was approved by the FDA in 2007 in combination with capecitabine and also has been shown to provide a benefit when combined with trastuzumab $[126,127]$. Additional monoclonal antibodies (pertuzumab, based on the CLEOPATRA study) [128] as well as ADCs (ado-trastuzumab emtansine (T-DM1) based on the EMILIA study, and famtrastuzumab deruxtecan (T-DXd) based on the DESTINY-Breast01 study) $[129,130]$ have entered practice in the metastatic setting either alone or in combination with trastuzumab. Additional TKIs (neratinib based on the NALA trial and tucatinib based on the HER2CLIMB study) $[131,132]$ have also been approved. Pertuzumab can be effective when combined with trastuzumab and chemotherapy in adjuvant and neoadjuvant settings [133,134]. As a whole, advances in targeted agents with improved efficacy, including superior intracranial activity [135], have greatly improved the prognosis for breast cancer expressing HER2.

In gastric cancer, trastuzumab was initially approved in the metastatic setting in 2010 based on the TOGA trial [136]. Most recently, T-DXd received FDA approval in the metastatic setting in January 2021 based on the DESTINY-gastric01 trial [137]. In this latter study, T-DXd was compared to chemotherapy for metastatic patients who had received two prior systemic lines of therapy, including trastuzumab. Both an improvement in ORR (51\% vs. $14 \%, p<0.001)$ and median OS were seen (12.5 vs. 8.4 months, HR 0.59). Approximately $10 \%$ of T-DXd treated patients developed interstitial lung disease or pneumonitis, although the majority were grades 1 or 2 .

\subsubsection{On-the Horizon: Colorectal Cancer}

While no FDA approvals have occurred to date, several clinical trials have evaluated HER2-directed agents, either alone or in combination, for advances colorectal cancer patients exhibiting HER2 overexpression. Combinatorial regimens include pertuzumab and trastuzumab in the MyPathway and TRIUMPH trials [138,139]; trastuzumab and lapatinib in HERACLES-A [140]; trastuzumab and tucatinib in MOUNTAINEER [141]; and T-DM1 and pertuzumab in HERACLES-B [142]. Additionally, data for T-DXd in DESTINYCRC01 was recently reported [143] Taken as a whole, these studies showed variable ORRs from $9 \%$ to $55 \%$ with seemingly better responses found for those patients whose tumors are KRAS wild-type. 


\subsubsection{On-the-Horizon: HER2 Mutations in NSCLC}

In addition to HER2 overexpression discussed in breast, gastric and colorectal cancer, activating HER2 mutations are found in multiple solid tumors and are potentially drugable [144], but no approvals have occurred to date. In NSCLC, driver HER2 mutations are identified in approximately $2 \%$ of patients. In a phase II basket trial utilizing T-DM1 in mostly pre-treated metastatic NSCLC patients, 8/18 (44\%) obtain a partial response [145]. Responses were seen in patients with HER2 exon 20 insertions as well as other kinase and non-kinase domain mutations. Additionally, Smit and colleagues reported data from the cohort of DESTINY-Lung01 which evaluated T-DXd in NSCLC patients with activating HER2 mutations, on which $90 \%$ were relegated to the kinase domain [146] With most patients previously receiving both chemotherapy and ICI, an impressive ORR of $62 \%$ $(26 / 42)$ was seen, for which the median duration was not reached at the time of data cutoff (median follow-up eight months).

\subsection{RET}

Medullary Thyroid, Other Thyroid and Lung Cancers

An additional predictive molecular target with multi-tumor approved agents are RET alterations, with potentially sensitizing mutations seen in approximately $70 \%$ of medullary thyroid cancers (MTC), fusions in $<10 \%$ of other thyroid cancers and fusions in $1-2 \%$ of NSCLC [147-149]. While 'dirty' multikinase inhibitors previously showed some activity in $R E T$-altered disease, a tradeoff of significant toxicity was seen. However, in May 2020 the FDA approved selpercatinib, a selective small-molecule RET inhibitor for adult NSCLC patients with metastatic RET fusions, as well as patients $\geq 12$ years old with $R E T$-mutated MTC or fusion-positive thyroid cancer who require systemic therapy (and are refractory to radioactive iodine, if indicated). Approval was based upon the results of LIBRETTO-001, a multicenter, open-label, multi-cohort trial. Key findings reported in RET fusion-positive NSCLC include a $64 \%$ ORR of 105 consecutively enrolled and pre-treated patients, with a median duration of almost 18 months; for untreated NSCLC patients, an $85 \%$ ORR was seen [150]. Importantly, only $2 \%$ discontinued selpercatinib due to drug-related toxicity, and $91 \%(n=11$ of 12$)$ with measurable central nervous system disease had an intracranial response. In RET-altered thyroid cancer, Wirth et al. detailed an ORR of $73 \%$ and $92 \%$ 1-year PFS of 88 untreated MTC patients harboring RET mutations; of 55 other MTC patients who received prior multi-kinase TKIs vandetinib and/or cabozantinib, impressive response rates and durability were still seen [151].

It should also be noted that pralsetinib was subsequently approved for RET fusions in advanced NSCLC in September 2020 and advanced, mutated or fusion-positive thyroid cancer patients (similar to the selpercatinib indication) in December 2020 based upon the ARROW trial. For NSCLC, a 57\% ORR of 87 previously-treated patients was observed; further, an additional 27 untreated fusion-positive NSCLC patients had a 70\% ORR to pralsetinib, with nearly $60 \%$ of responses extending beyond six months $[37,152,153]$. Clinical progression to selpercatinib (or pralsetinib) should prompt molecular testing for resistance mechanisms, which can include RET solvent front mutations, as well as MET and KRAS amplifications [154,155]. While some resistance mechanisms may be targetable, development of next-generation RET inhibitors is warranted for solvent front alterations.

\subsection{DNA Damage Response/PARP Inhibition}

Many loss-of-function alterations occur in genes involved in DNA repair, particularly in homologous recombination repair (HRR). BRCA1 and BRCA2 are the most common and most well-characterized genes involved in this process, but other genes such as ATM, CHEK2, PALB2 and RAD51 are also linked to HRR deficiency (HRD). Deleterious mutations in HRR can be found both in the germline setting as well as somatic alterations uncovered with tumor molecular profiling. Responses to poly(adenosine diphosphateribose) polymerase (PARP) inhibition have been described in several tumor types with 
HRD, either by specific mutations identified in a candidate gene or via genomic instability identified during molecular profiling.

\subsubsection{Ovarian Cancer}

Ovarian cancer is known to be associated with germline alterations in BRCA1 or BRCA2, though somatic mutations can also occur. Olaparib was approved in 2018 for the maintenance of patients with somatic mutations in the setting of complete or partial response to first-line platinum therapy. This indication is based on the SOLO-1 trial comparing olaparib versus placebo in this setting. Progression-free survival was improved in the olaparib arm (HR 0.30; $p<0.0001)$ [156]. The indication was expanded to include a combination of olaparib with bevacizumab in 2020 based on the PAOLA-1 trial [157]. The PARP inhibitor rucaparib initially had approval only in patients with BRCA germline mutations. However, soon thereafter rucaparib was approved in the maintenance setting for ovarian cancer regardless of BRCA status. Interestingly, the PARP inhibitor rucaparib was later approved regardless of BRCA status based on the ARIEL3 trial. While this trial evaluated patients for BRCA or HRD, the overall study population showed a benefit in PFS (HR 0.36, $p$ < 0.0001) [158]. In 2017, approval for the PARP inhibitor niraparib was obtained for maintenance therapy in ovarian cancer, also without the need for HRD mutations with this indication expanded to the first-line maintenance setting in 2020 based on the PRIMA trial [159]. In 2019, however, niraparib was approved again in late-line ovarian cancer for patients with HRD mutations [42]. As is clear from these approval timelines, PARP inhibitor therapy in ovarian cancer remains complex, with certain agents approved in certain settings regardless of genomic alterations, some approved only for BRCA1/2 genomic alterations and others approved for germline or somatic alterations in BRCA1/2 or HRD tumors.

\subsubsection{Prostate Cancer}

The approval of PARP inhibitors for somatic HRD mutations in prostate cancer was based on the PROfound study which randomized 256 patients to the PARP inhibitor olaparib and 131 patients to the investigator's choice of hormone therapy. Patients were divided into two cohorts, one with mutations in BRCA1, BRCA2 or ATM and the other with mutations among 12 other genes in the HRR pathway. A statistically significant difference was seen in PFS among the BRCA/ATM cohort (HR 0.34, $p<0.0001$ ) as well as overall across both cohorts (HR 0.49; $p<0.0001$ ) [160]. Even more recently, the PARP inhibitor rucaparib was also approved in castrate-resistant prostate cancer in patients specifically with BRCA1 or two mutations, whether germline or somatic, who have failed hormone therapy and a taxane. The approval was based on the TRITON2 trial, a single-arm trial of 115 patients showing a confirmed objective response rate of $44 \%$ among the 65 patients who had measurable disease [161].

\subsubsection{Breast Cancer}

In January 2018, the FDA approved olaparib in metastatic HER-2 negative breast cancer patients whose tumors harbored a germline $B R C A$ mutation and received prior chemotherapy based upon the results of the randomized phase 3 OlympiAD trial [162]. In this study, olaparib was found to have an ORR of $60 \%$ and a superior PFS compared to physician's choice of chemotherapy (7.0 versus 3.2 months; HR 0.58). Subsequently, talazoparib received a similar approval for germline $B R C A$ mutated metastatic breast cancer, with the EMBRACA trial demonstrating a higher ORR (63\% versus $27 \%, p<0.001)$ and improved median PFS (8.6 versus 5.6 months; HR 0.54) when compared to chemotherapy [163].

\subsubsection{Pancreatic Cancer}

Germline BRCA1/2 mutations occur in approximately $5 \%$ of pancreatic cancers [164]. On 27 December 2019, olaparib was FDA approved for this subset of metastatic pancreatic 
cancer patients in the maintenance setting, where the agent was given after a minimum of 16 weeks of platinum-based chemotherapy. Golan and colleagues reported an improvement in median PFS (7.4 versus 3.8 months for placebo; HR 0.53); however, an interim analysis did not show a difference in OS [165].

A summary of major clinical trials leading to approvals in more than one malignancy can be seen in Table 2.

\subsection{PD-L1}

The use of ICI therapy, particularly anti-programmed death-1 (PD1) antibodies or anti-programmed death ligand-1 (PD-L1) antibodies, has become virtually ubiquitous in cancer. Currently, there are seven anti-PD-1 or PD-L1 therapies in use in the clinic, approved across 19 malignancies and with 77 different indications [166-172]. Being as the mechanism of action of these therapies depends upon the interaction between PD-1 and PDL1, PD-L1 protein expression was early determined to be a potential predictor of response to ICI [173]. However, the fact that some tumors with high expression of PD-L1 do not respond to PD-(L)1 therapy, and the fact that some tumors with no or low PD-L1 expression do respond to PD-(L)1 therapy, highlights the difficulty in using this biomarker as a true surrogate of response [173]. Additionally, various companion diagnostics using different detection antibodies, methods of measuring PD-L1 expression and "positive" cutoffs have been problematic and present a barrier to the interpretation of biomarker data in clinical trials [174]. In an evaluation of the pivotal trials leading to 45 FDA approvals of PD-(L)1 inhibitors from 2015-2019, PD-L1 expression was predictive in only 29\% of the approvals, while it was not predictive in 53\% and not tested in 18\% [175]. However, while PD-L1 expression is not always predictive, failure to include the biomarker into certain clinical trials or utilization of the wrong assay or wrong cutoff may have led to a determination of an overall lack of efficacy $[176,177]$. At the current time, 12 indications for PD-(L)1 therapy in seven malignancies are dependent on PD-L1 status [166-168,171]. These indications utilize different methods of determining PD-L1 status (tumor cell proportion score, immune cell proportion score or combined positive score), different thresholds for positivity and different FDA-approved companion diagnostics. Table 3 summarizes the PD-L1 based approvals that exist in lung, head and neck, bladder, gastric, esophageal, cervical and breast cancer together with the various measures of PD-L1 expression, companion diagnostics and positive thresholds. In summary, PD-L1 remains a highly imperfect biomarker, and other markers of immune responsiveness are simultaneously being tested for (e.g., MSI status, TMB-h) and studied (tumor-infiltrating lymphocytes, tumor microenvironment, etc.) to enable proper selection of treatment with ICIs [174]. 
Table 2. Key pivotal trials leading to FDA approval in alterations with multiple tumor indications (MSI-h, TMB-h, NTRK fusion, BRAF, HER2, RET, BRCA).

\begin{tabular}{|c|c|c|c|c|c|c|c|c|c|c|c|}
\hline $\begin{array}{l}\text { Setting/Genomic } \\
\text { Alteration }\end{array}$ & $\begin{array}{l}\text { Cancer Type, } \\
\text { Line of Therapy }\end{array}$ & Study & Trial Phase & $\begin{array}{l}\text { Number of } \\
\text { Subjects }\end{array}$ & Line of Therapy & Agent & Comparator & $\begin{array}{l}\text { Primary } \\
\text { Outcome }\end{array}$ & $\begin{array}{l}\text { Primary } \\
\text { Outcome } \\
\text { Results }\end{array}$ & $\begin{array}{l}\text { Key Secondary } \\
\text { Outcomes }\end{array}$ & $\begin{array}{l}\text { Results (If } \\
\text { Applicable) }\end{array}$ \\
\hline \multirow{2}{*}{ MSI-h, dMMR } & Pan-tumor & $\underset{[51]}{\text { KEYNOTE-158 }}$ & II & 233 & $\begin{array}{l}\text { Metastatic } 2 n d \\
\text { line or greater }\end{array}$ & Pembrolizumab & None & $\begin{array}{l}\text { ORR (objective } \\
\text { response rate) }\end{array}$ & $\begin{array}{l}34.3 \%(95 \% \mathrm{CI}, \\
28.3 \%, 40.8 \%)\end{array}$ & $\begin{array}{l}\text { Overall survival } \\
\text { (OS) }\end{array}$ & $\begin{array}{l}\text { 23.5 mo }(95 \% \mathrm{CI}, \\
\text { 13.5-not reached } \\
\text { (NR)) }\end{array}$ \\
\hline & $\begin{array}{l}\text { Colorectal } \\
\text { cancer }\end{array}$ & $\begin{array}{c}\text { KEYNOTE-177 } \\
{[54]}\end{array}$ & III & 307 & $\begin{array}{l}\text { Metastatic 1st } \\
\text { line }\end{array}$ & Pembrolizumab & Chemotherapy & $\begin{array}{l}\text { Progression-free } \\
\text { survival (PFS) } \\
\text { (median) }\end{array}$ & $\begin{array}{c}16.5 \mathrm{v} 8.2 \\
\text { months, hazard } \\
\text { ratio (HR) } 0.60 \\
(95 \% \text { CI, } \\
0.45-0.8, p \\
0.0004)\end{array}$ & ORR & $44 \%$ vs. $33 \%$ \\
\hline TMB-high & Pan-tumor & $\begin{array}{c}\text { KEYNOTE-158 } \\
\text { [65] }\end{array}$ & II & 102 & $\begin{array}{l}\text { Metastatic 2nd } \\
\text { line or greater }\end{array}$ & Pembrolizumab & $\begin{array}{l}\text { TMB-low } \\
\text { patients } \\
(\mathrm{n}=688)\end{array}$ & ORR & $\begin{array}{c}29 \%(95 \% \text { CI, } \\
21-39 \%) \text { vs. } 6 \% \\
(95 \% \text { CI, } 5-8 \%) \\
\end{array}$ & N/A & N/A \\
\hline \multirow{2}{*}{ NTRK fusion } & \multirow{2}{*}{ Pan-tumor } & $\begin{array}{c}\text { LOXO-TRK-14001, } \\
\text { SCOUT, NAVIGATE } \\
\text { (pooled analysis) } \\
\text { [59] }\end{array}$ & $\mathrm{I} / \mathrm{II}$ & 159 & Any metastatic & Larotrectinib & None & ORR & $\begin{array}{c}79 \%(95 \% \text { CI } \\
72-85 \%)\end{array}$ & $\begin{array}{l}\text { Duration of } \\
\text { response (DOR) } \\
\text { (median) }\end{array}$ & $\begin{array}{c}35.2 \mathrm{mo}(95 \% \mathrm{CI} \\
22.8-\mathrm{NR})\end{array}$ \\
\hline & & $\begin{array}{c}\text { ALKA-372-001, } \\
\text { STARTRK-1, STARTRK-2 } \\
\text { (pooled analysis) } \\
\text { [60] }\end{array}$ & $\mathrm{I} / \mathrm{II}$ & 54 & Any metastatic & Entrectinib & None & ORR & $\begin{array}{c}57 \%(95 \% \mathrm{CI} \\
43-71 \%)\end{array}$ & DOR (median) & $\begin{array}{c}10 \text { mo }(95 \% \text { CI, } \\
7.1-\text { not } \\
\text { estimable (NE)) }\end{array}$ \\
\hline \multirow{5}{*}{ BRAF V600E } & \multirow[t]{3}{*}{ Melanoma } & $\begin{array}{c}\text { CoBRIM } \\
{[85]}\end{array}$ & III & 495 & $\begin{array}{l}\text { Metastatic 1st } \\
\text { line }\end{array}$ & $\begin{array}{l}\text { Vemurafenib } \\
\text { and } \\
\text { Cobimetinib }\end{array}$ & Vemurafenib & PFS (median) & $\begin{array}{c}12.3 \text { v } 7.2 \\
\text { months HR } 0.58 \\
(95 \% \text { CI, } \\
0.46-0.72 \\
p=<0.0001)\end{array}$ & OS (median) & $\begin{array}{c}22.3 \text { v } 17.4 \\
\text { months HR } 0.70 \\
(95 \% \text { CI, } \\
0.55-0.90 \\
p=0.005)\end{array}$ \\
\hline & & $\begin{array}{l}\text { COLUMBUS } \\
{[87]}\end{array}$ & III & 383 & $\begin{array}{l}\text { Metastatic 2nd } \\
\text { line or greater }\end{array}$ & $\begin{array}{l}\text { Encorafenib } \\
\text { and } \\
\text { Binimetinib }\end{array}$ & Vemurafenib & PFS (median) & $\begin{array}{c}14.9 \text { v } 7.3 \\
\text { months HR } 0.54 \\
(95 \% \text { CI, } \\
0.41-0.71 \\
p<0.0001)\end{array}$ & OS (median) & $\begin{array}{c}33.6 \mathrm{v} 16.9 \\
\text { months HR } 0.61 \\
(95 \% \text { CI, } \\
0.48-0.79 \\
p<0.0001)\end{array}$ \\
\hline & & $\underset{[90]}{\text { COMBI-AD }}$ & III & 870 & $\begin{array}{l}\text { Stage III } \\
\text { adjuvant }\end{array}$ & $\begin{array}{l}\text { Dabrafenib } \\
\text { and } \\
\text { Trametinib }\end{array}$ & Placebo & $\begin{array}{l}\text { Relapse-free } \\
\text { survival (RFS) } \\
\text { (three-year) }\end{array}$ & $\begin{array}{c}58 \% \text { vs. } 39 \% \text { HR } \\
0.47(95 \% \text { CI, } \\
0.39-0.58, p< \\
0.001)\end{array}$ & OS (three-year) & $\begin{array}{c}86 \% \text { vs. } 77 \%, \mathrm{HR} \\
0.57(95 \% \mathrm{CI}, \\
0.42-0.79, \\
p=0.0006)\end{array}$ \\
\hline & NSCLC & $\begin{array}{c}\text { BRF113928 } \\
{[105]}\end{array}$ & II & 36 & $\begin{array}{l}\text { Metastatic 1st } \\
\text { line }\end{array}$ & $\begin{array}{l}\text { Dabrafenib } \\
\text { and } \\
\text { Trametinib }\end{array}$ & None & ORR & $\begin{array}{c}64 \%(95 \% \mathrm{CI} \\
46-79 \%)\end{array}$ & N/A & N/A \\
\hline & $\begin{array}{l}\text { Anaplastic } \\
\text { thyroid cancer }\end{array}$ & $\underset{[113]}{\text { CDRB436 }} \times 2201$ & II & 16 & $\begin{array}{c}\text { Any line } \\
\text { post-radiation or } \\
\text { surgery }\end{array}$ & $\begin{array}{l}\text { Dabrafenib } \\
\text { and } \\
\text { Trametinib }\end{array}$ & None & ORR & $\begin{array}{c}69 \%(95 \% \mathrm{CI} \\
41-89 \%)\end{array}$ & DOR (median) & Not reached \\
\hline
\end{tabular}


Table 2. Cont.

\begin{tabular}{|c|c|c|c|c|c|c|c|c|c|c|c|}
\hline $\begin{array}{l}\text { Setting/Genomic } \\
\text { Alteration }\end{array}$ & $\begin{array}{l}\text { Cancer Type, } \\
\text { Line of Therapy }\end{array}$ & Study & Trial Phase & $\begin{array}{c}\text { Number of } \\
\text { Subjects }\end{array}$ & Line of Therapy & Agent & Comparator & $\begin{array}{l}\text { Primary } \\
\text { Outcome }\end{array}$ & $\begin{array}{c}\text { Primary } \\
\text { Outcome } \\
\text { Results }\end{array}$ & $\begin{array}{l}\text { Key Secondary } \\
\text { Outcomes }\end{array}$ & $\begin{array}{l}\text { Results (If } \\
\text { Applicable) }\end{array}$ \\
\hline & $\begin{array}{l}\text { Colorectal } \\
\text { cancer }\end{array}$ & $\underset{[114]}{\text { BEACON-CRC }}$ & III & 665 & $\begin{array}{l}\text { Metastatic 2nd } \\
\text { line or greater }\end{array}$ & $\begin{array}{l}\text { Encorafenib, } \\
\text { Binimetinib } \\
\text { and } \\
\text { Cetuximab }\end{array}$ & $\begin{array}{l}\text { Investigator } \\
\text { choice }\end{array}$ & OS (median) & $\begin{array}{c}9.0 \text { mo vs. } 5.4 \\
\text { mo, HR } 0.52 \\
(95 \% \mathrm{CI}, \\
0.39-0.70, p< \\
0.001)\end{array}$ & ORR & $\begin{array}{c}26 \%(95 \% \text { CI } \\
18-35 \%) \text { vs. } 2 \% \\
(95 \% \text { CI, } 0-7 \%)\end{array}$ \\
\hline \multirow{8}{*}{ HER2 positive } & \multirow{6}{*}{ Breast cancer } & $\begin{array}{c}\text { Slamon et al. (2001) } \\
\text { [7] }\end{array}$ & III & 469 & $\begin{array}{l}\text { Metastatic 1st } \\
\text { line }\end{array}$ & $\begin{array}{l}\text { Trastuzumab } \\
\text { and } \\
\text { chemotherapy }\end{array}$ & $\begin{array}{l}\text { Placebo and } \\
\text { chemotherapy }\end{array}$ & PFS (median) & $\begin{array}{c}7.4 \text { mo vs. } 4.6 \\
\text { mo, HR } 0.51 \\
(95 \% \mathrm{CI} \\
0.41-0.63 \\
p<0.001) \\
\end{array}$ & OS (median) & $\begin{array}{c}25.1 \text { mo vs. } 20.3 \\
\text { mo, HR } 0.80 \\
(95 \% \text { CI } \\
0.64-1.00 \\
p=0.046)\end{array}$ \\
\hline & & $\begin{array}{l}\text { EMILIA } \\
{[130]}\end{array}$ & III & 991 & $\begin{array}{l}\text { Metastatic 2nd } \\
\text { line or greater }\end{array}$ & $\begin{array}{c}\text { Trastuzumab } \\
\text { emtansine } \\
\text { (T-DM1) }\end{array}$ & $\begin{array}{l}\text { Lapatinib and } \\
\text { Capecitabine }\end{array}$ & PFS (median) & $\begin{array}{c}9.6 \text { mo vs. } 6.4 \\
\text { mo, HR } 0.65 \\
(95 \% \text { CI, } \\
0.55-0.77 \\
p<0.001)\end{array}$ & OS (median) & $\begin{array}{c}30.9 \text { mo vs. } 25.1 \\
\text { mo, HR } 0.68 \\
(95 \% \text { CI, } \\
0.55-0.85 \\
p<0.001)\end{array}$ \\
\hline & & $\begin{array}{c}\text { DESTINY-Breast01 } \\
{[129]}\end{array}$ & II & 184 & $\begin{array}{l}\text { Metastatic 3rd } \\
\text { line or greater }\end{array}$ & $\begin{array}{c}\text { Trastuzumab } \\
\text { deruxtecan } \\
\text { (T-DXd) }\end{array}$ & None & ORR & $\begin{array}{c}60.9 \%(95 \% \mathrm{CI} \\
53-68 \%)\end{array}$ & PFS & $\begin{array}{c}16.4 \mathrm{mo}(95 \% \mathrm{CI}, \\
12.7-\mathrm{NR})\end{array}$ \\
\hline & & $\begin{array}{c}\text { CLEOPATRA } \\
{[128]}\end{array}$ & III & 808 & $\begin{array}{l}\text { Metastatic 1st } \\
\text { line }\end{array}$ & $\begin{array}{l}\text { Pertuzumab, } \\
\text { trastuzumab } \\
\text { and docetaxel }\end{array}$ & $\begin{array}{l}\text { Trastuzumab } \\
\text { and docetaxel }\end{array}$ & PFS (median) & $\begin{array}{c}18.5 \text { mo vs. } 12.4 \\
\text { mo, HR } 0.62 \\
\text { (95\% } \mathrm{CI}, \\
0.51-0.75 \\
p<0.001)\end{array}$ & OS & $\begin{array}{c}\text { HR } 0.64(95 \% \text { CI, } \\
0.47-0.88, \\
p=0.005)\end{array}$ \\
\hline & & $\begin{array}{l}\text { NALA } \\
{[131]}\end{array}$ & III & 621 & $\begin{array}{l}\text { Metastatic 2nd } \\
\text { line or greater }\end{array}$ & $\begin{array}{l}\text { Neratinib and } \\
\text { capecitabine }\end{array}$ & $\begin{array}{l}\text { Lapatinib and } \\
\text { Capecitabine }\end{array}$ & PFS & $\begin{array}{c}\text { HR } 0.76(95 \% \text { CI } \\
0.63-0.93 \\
p=0.0059)\end{array}$ & $\begin{array}{l}\text { OS (co-primary } \\
\text { endpoint) }\end{array}$ & $\begin{array}{c}\text { HR } 0.88(95 \% \mathrm{CI}, \\
0.72-1.07, \\
p=0.21)\end{array}$ \\
\hline & & $\begin{array}{l}\text { HER2CLIMB } \\
\text { [132] }\end{array}$ & II & 612 & $\begin{array}{l}\text { Metastatic 3rd } \\
\text { line or greater }\end{array}$ & $\begin{array}{l}\text { Tucatinib, } \\
\text { trastuzumab } \\
\text { and } \\
\text { capecitabine }\end{array}$ & $\begin{array}{l}\text { Trastuzumab } \\
\text { and capecitabine }\end{array}$ & PFS (median) & $\begin{array}{c}7.8 \text { mo vs. } 5.6 \\
\text { mo, HR } 0.54 \\
\text { (95\% CI, } \\
0.42-0.71 \\
p<0.001)\end{array}$ & OS (median) & $\begin{array}{c}21.9 \text { mo vs. } 17.4 \\
\text { mo, HR } 0.66 \\
(95 \% \mathrm{CI} \\
0.50-0.88 \\
p=0.005)\end{array}$ \\
\hline & \multirow{2}{*}{ Gastric cancer } & $\begin{array}{l}\text { TOGA } \\
\text { [136] }\end{array}$ & III & 594 & $\begin{array}{l}\text { Metastatic 1st } \\
\text { line }\end{array}$ & $\begin{array}{c}\text { Trastuzumab } \\
\text { and } \\
\text { Chemother- } \\
\text { apy }\end{array}$ & Chemotherapy & OS (median) & $\begin{array}{c}13.8 \text { mo vs. } 11.1 \\
\text { mo, HR } 0.74 \\
\text { (95\% } \mathrm{CI}, \\
0.60-0.91 \\
p=0.0046)\end{array}$ & N/A & N/A \\
\hline & & $\begin{array}{c}\text { DESTINY-Gastric01 } \\
{[137]}\end{array}$ & II & 187 & $\begin{array}{l}\text { Metastatic 3rd } \\
\text { line or greater }\end{array}$ & T-DXd & Chemotherapy & ORR & $\begin{array}{c}51 \% \text { vs. } 14 \% \\
(p<0.001)\end{array}$ & OS (median) & $\begin{array}{c}12.5 \text { mo vs. } 8.4 \\
\text { mo, } \mathrm{HR} 0.59 \\
(95 \% \mathrm{CI} \\
0.39-0.88 \\
p=0.01)\end{array}$ \\
\hline RET fusion & NSCLC & $\begin{array}{c}\text { LIBRETTO-001 } \\
\text { [150] }\end{array}$ & $\mathrm{I} / \mathrm{II}$ & 105 & $\begin{array}{l}\text { Metastatic } \\
\text { previously } \\
\text { treated }\end{array}$ & Selpercatinib & None & ORR & $\begin{array}{l}64 \%(95 \% \text { CI } \\
54-73 \%)\end{array}$ & DOR (median) & $\begin{array}{c}17.5 \text { mo }(95 \% \mathrm{CI}, \\
12.0-\mathrm{NE})\end{array}$ \\
\hline
\end{tabular}


Table 2. Cont.

\begin{tabular}{|c|c|c|c|c|c|c|c|c|c|c|c|}
\hline $\begin{array}{l}\text { Setting/Genomic } \\
\text { Alteration }\end{array}$ & $\begin{array}{l}\text { Cancer Type, } \\
\text { Line of Therapy }\end{array}$ & Study & Trial Phase & $\begin{array}{l}\text { Number of } \\
\text { Subjects }\end{array}$ & Line of Therapy & Agent & Comparator & $\begin{array}{l}\text { Primary } \\
\text { Outcome }\end{array}$ & $\begin{array}{l}\text { Primary } \\
\text { Outcome } \\
\text { Results }\end{array}$ & $\begin{array}{l}\text { Key Secondary } \\
\text { Outcomes }\end{array}$ & $\begin{array}{l}\text { Results (If } \\
\text { Applicable) }\end{array}$ \\
\hline & & $\begin{array}{c}\text { ARROW } \\
\text { [153] }\end{array}$ & $\mathrm{I} / \mathrm{II}$ & 87 & $\begin{array}{l}\text { Metastatic } \\
\text { previously } \\
\text { treated }\end{array}$ & Pralsetinib & None & ORR & $\begin{array}{c}57 \%(95 \% \text { CI } \\
46-68 \%)\end{array}$ & $\mathrm{N} / \mathrm{A}$ & N/A \\
\hline RET mutation & $\begin{array}{c}\text { Medullary } \\
\text { thyroid cancer }\end{array}$ & $\begin{array}{c}\text { LIBRETTO-001 } \\
\text { [151] }\end{array}$ & $\mathrm{I} / \mathrm{II}$ & 88 & $\begin{array}{l}\text { Metastatic 1st } \\
\text { line }\end{array}$ & Selpercatinib & None & ORR & $\begin{array}{c}73 \% \text { (95\% CI, } \\
62-82 \%\end{array}$ & N/A & $\mathrm{N} / \mathrm{A}$ \\
\hline \multirow{4}{*}{$\begin{array}{l}\text { BRCA or HRD } \\
\text { alteration }\end{array}$} & Ovarian cancer & $\begin{array}{c}\text { SOLO-1 } \\
{[156]}\end{array}$ & III & 391 & $\begin{array}{l}\text { Metastatic 1st } \\
\text { line } \\
\text { maintenance }\end{array}$ & Olaparib & Placebo & PFS (-three-year) & $\begin{array}{c}60 \% \text { vs. } 27 \%, \mathrm{HR} \\
0.30(95 \% \mathrm{CI} \\
0.23-0.41, p< \\
0.001)\end{array}$ & N/A & $\mathrm{N} / \mathrm{A}$ \\
\hline & $\begin{array}{l}\text { Castrate- } \\
\text { resistant } \\
\text { prostate cancer }\end{array}$ & $\underset{\text { [161] }}{\text { TRITON2 }}$ & II & 115 & $\begin{array}{l}\text { Metastatic, post } \\
\text { androgen and } \\
\text { chemotherapy }\end{array}$ & Rucaparib & None & ORR & $\begin{array}{l}43.5 \%(95 \% \mathrm{CI}, \\
31.0-56.7 \%)\end{array}$ & $\begin{array}{l}\text { Prostate-specific } \\
\text { antigen response }\end{array}$ & $\begin{array}{c}54.8 \%(95 \% \mathrm{CI} \\
45.2-64.1 \%)\end{array}$ \\
\hline & Breast cancer & $\begin{array}{l}\text { OlympiAD } \\
\text { [162] }\end{array}$ & III & 301 & $\begin{array}{l}\text { Metastatic, } \\
\text { germline, no } \\
\text { more than two } \\
\text { prior lines }\end{array}$ & Olaparib & Chemotherapy & PFS (median) & $\begin{array}{c}7.0 \text { vs. } 4.2 \mathrm{mo}, \\
\text { HR } 0.58(95 \% \mathrm{CI}, \\
0.43-0.80, p= \\
0.0009)\end{array}$ & OS (median) & $\begin{array}{c}19.3 \text { vs. } 17.1 \mathrm{mo}, \\
\text { HR } 0.90(95 \% \mathrm{CI}, \\
0.66-1.23)\end{array}$ \\
\hline & $\begin{array}{l}\text { Pancreatic } \\
\text { cancer }\end{array}$ & $\begin{array}{c}\text { POLO } \\
\text { [165] }\end{array}$ & III & 154 & $\begin{array}{l}\text { Metastatic, } \\
\text { germline, 1st } \\
\text { line } \\
\text { maintenance }\end{array}$ & Olaparib & Placebo & PFS (median) & $\begin{array}{c}\text { 7.4 vs. } 3.8 \mathrm{mo}, \\
\text { HR 0.53 }(95 \% \text { CI } \\
0.35-0.81, p= \\
0.0035)\end{array}$ & ORR & $23 \%$ vs. $12 \%$ \\
\hline
\end{tabular}


Table 3. Indications for use of PD-1 or PD-L1 antibodies dependent on PD-L1 level.

\begin{tabular}{|c|c|c|c|c|c|c|}
\hline Malignancy & Line & Agent & Measurement & $\begin{array}{l}\text { Positive } \\
\text { Threshold }\end{array}$ & $\begin{array}{l}\text { FDA-Approved } \\
\text { Companion } \\
\text { Diagnostic }\end{array}$ & FDA Approval Date \\
\hline \multirow{5}{*}{ Lung cancer } & \multirow{5}{*}{ 1st line metastatic } & Pembrolizumab & $\begin{array}{l}\text { Tumor proportion } \\
\text { score (TPS) }\end{array}$ & $>1 \%$ & 22c3 Ab, Dako & 11 March 2019 [178] \\
\hline & & $\begin{array}{l}\text { Nivolumab + } \\
\text { Ipilimumab }\end{array}$ & TPS & $>1 \%$ & 28-8 Ab, Dako & 15 May 2020 [179] \\
\hline & & \multirow{2}{*}{ Atezolizumab } & $\begin{array}{l}\text { Tumor cell proportion } \\
\text { score (TC) }\end{array}$ & $>50 \%$ & SP142 Ab, Ventana & \multirow[t]{2}{*}{18 May 2020 [180] } \\
\hline & & & $\begin{array}{c}\text { Immune cell } \\
\text { proportion score (IC) }\end{array}$ & $>10 \%$ & SP142 Ab, Ventana & \\
\hline & & Cemiplimab & TPS & $>50 \%$ & 22c3 Ab, Dako & 22 February 2021 [181] \\
\hline $\begin{array}{l}\text { Head and neck } \\
\text { cancer }\end{array}$ & 1st line metastatic & Pembrolizumab & $\begin{array}{c}\mathrm{TC}+\mathrm{IC} \text { (combined } \\
\text { positive score or CPS) }\end{array}$ & $>1$ & 22c3 Ab, Dako & 10 June 2019 [182] \\
\hline \multirow{2}{*}{ Bladder cancer } & \multirow{2}{*}{$\begin{array}{l}\text { 1st line metastatic } \\
\text { cisplatin ineligible }\end{array}$} & Pembrolizumab & CPS & $>10$ & 22c3 Ab, Dako & 19 June 2018 [183] \\
\hline & & Atezolizumab & IC & $>5 \%$ & SP142 Ab, Ventana & 19 June 2018 [183] \\
\hline Gastric cancer & $\begin{array}{l}>3 \text { rd line } \\
\text { metastatic }\end{array}$ & Pembrolizumab & CPS & $>1$ & 22c3 Ab, Dako & 22 September 2017 [184] \\
\hline $\begin{array}{l}\text { Esophageal } \\
\text { cancer } \\
\text { (squamous) }\end{array}$ & $\begin{array}{l}>3 \text { rd line } \\
\text { metastatic }\end{array}$ & Pembrolizumab & CPS & $>10$ & 22c3 Ab, Dako & 30 July 2019 [185] \\
\hline Cervical cancer & $\begin{array}{l}>2 \text { nd line } \\
\text { metastatic }\end{array}$ & Pembrolizumab & CPS & $>1$ & 22c3 Ab, Dako & 12 June 2018 [186] \\
\hline \multirow{2}{*}{$\begin{array}{c}\text { Breast cancer } \\
\text { (triple negative) }\end{array}$} & \multirow{2}{*}{ Metastatic } & Atezolizumab & IC & $>1 \%$ & SP142 Ab, Ventana & 8 March 2019 [187] \\
\hline & & Pembrolizumab & CPS & $>10$ & 22c3 Ab, Dako & 13 November 2020 [188] \\
\hline
\end{tabular}

\subsection{On the Horizon-KRAS G12C}

$K R A S$, which controls cellular signal transduction through its encoded guanosine triphosphatase activity, is the most commonly mutated oncogene in solid tumors, frequently portends a poor prognosis, is affiliated with resistance to multiple systemic treatments and thus far, its targeting has remained elusive [189-192]. Occurring in approximately $13 \%$ of NSCLC and $>1 \%$ of colorectal cancer and various other solid tumors, KRAS G12C mutations were found to be targetable in pre-clinical studies through irreversible, covalent binding of small molecule kinase inhibitors to the mutated cysteine and nearby P2 pocket of the switch II region [193-195]. Hong et al. recently reported phase 1 data, showing promising activity of KRAS G12C inhibitor, sotorasib, in 129 pre-treated (median number of prior lines of therapy was 3) solid tumor patients [196]. For example, $32 \%$ of NSCLC, $7 \%$ of colorectal cancer and $14 \%$ of other solid tumor patients (including melanoma, endometrial, pancreatic and appendiceal) had an objective response. While the median PFS of responders was six months with single-agent sotorasib, future evaluation of KRAS inhibitors with tumor-informed precision combinations may lead to more effective targeting [197-199]. Furthermore, the efficacy of a second covalent inhibitor of KRAS G12C, adagrasib, was presented at the European Lung Cancer Virtual Conference in early 2021, where the multicohort phase I/II trial showed a $45 \%$ ORR in 51 advanced, typically pre-treated NSCLC patients [200].

\section{Precision Oncology in Lung Cancer}

Perhaps more than any other solid tumor, patients with lung cancer can derive benefit from therapeutic options exposed following comprehensive molecular profiling. The leading cause of cancer-related mortality worldwide [201], advanced lung cancer treated with a one-size-fits-all approach of platinum doublet chemotherapy historically resulted in relatively poor outcomes, with a limited percentage of patients achieving long-term survival [202]. However, recent evidence showed a population-level reduction in mortality of lung cancer patients from 2013-2016, attributed not only to a reduction in incidence but an early indication of the efficacy of novel precision oncology treatments in NSCLC [203]. 
NSCLC accounts for approximately $85 \%$ of all lung cancer; its three major histologic types include adenocarcinoma, squamous cell carcinoma and large cell carcinoma. The vast majority of lung adenocarcinoma is driven by identifiable oncogenic aberrations, with a growing number amenable to targeted therapies such that current guidelines recommend complete molecular testing for patients with metastatic disease [204]. Additionally, testing should be considered for non-adenocarcinoma NSCLC, especially those with limited smoking history or mixed histology whose samples may be enriched for targetable mutations or alterations. While testing may take several forms, up-front comprehensive molecular profiling in NSCLC should ideally consist of a broad-panel evaluation such as NGS for specific gene mutations, IHC, ISH, real-time PCR and RNA assessment to identify gene rearrangements and fusions, as well as immune biomarker appraisal with IHC for PD-L1, MSI status and TMB (see previous discussion) [205]. If available tissue is not sufficient to complete testing, liquid biopsy with plasma cell-free or circulating tumor DNA can be informative $[206,207]$. Unless an impending clinical scenario mandates, the treatment team should ideally wait upon receipt of comprehensive molecular data to determine if the NSCLC patient is a candidate for first-line targeted therapy. The determination of whether to begin first-line treatment with ICI or targeted therapy is of great importance, as improper initial ICI in NSCLC patients with specific oncogenic drivers can lead to significant toxicity when subsequent TKIs or targeted therapy are begun $[208,209]$. In addition to the aforementioned targetable alterations (BRAF V600E, RET fusions, as well as emerging HER2 mutations and KRAS G12C), multiple other predictive molecular targets exist in NSCLC. Table 4 shows molecular alterations and their targeted agents that have been approved only in lung cancer.

Table 4. Molecular alterations leading to FDA-targeted therapy approvals only in NSCLC.

\begin{tabular}{|c|c|c|c|c|}
\hline Alteration(s) & Line of Therapy & Medications & Drug Class & FDA Approval Date \\
\hline \multirow{6}{*}{$\begin{array}{c}\text { EGFR } \\
\text { (exon } 19 \text { deletions and } \\
\text { exon } 21 \text { point } \\
\text { mutations) }\end{array}$} & \multirow{5}{*}{ 1st line metastatic } & Erlotinib & \multirow{6}{*}{ EGFR TKI } & 14 May 2013 [210] \\
\hline & & Gefitinib & & 13 July 2015 [211] \\
\hline & & Afatinib & & 12 January 2018 [212] \\
\hline & & Dacomitinib & & 27 September 2018 [213] \\
\hline & & $\begin{array}{c}\text { Osimertinib (also } \\
\text { against T790M } \\
\text { mutations) }\end{array}$ & & 18 April 2018 [214] \\
\hline & Adjuvant & $\begin{array}{c}\text { Osimertinib (also } \\
\text { against T790M } \\
\text { mutations) }\end{array}$ & & 18 December 2020 [215] \\
\hline $\begin{array}{c}\text { EGFR (exon } 20 \\
\text { insertion) }\end{array}$ & 2nd line metastatic & Amivantamab & $\begin{array}{c}\text { EGFR, MET bispecific } \\
\text { antibody }\end{array}$ & 21 May 2021 [216] \\
\hline \multirow{5}{*}{ ALK fusions } & \multirow{5}{*}{ Metastatic } & Crizotinib & \multirow{5}{*}{ ALK TKI } & 26 August 2011 [217] \\
\hline & & Ceritinib & & 29 April 2014 [218] \\
\hline & & Lorlatinib & & 2 November 2018 [219] \\
\hline & & Alectinib & & 11 December 2015 [220] \\
\hline & & Brigatinib & & 28 April 2017 [221] \\
\hline \multirow{2}{*}{ ROS1 fusions } & \multirow{2}{*}{ Metastatic } & Crizotinib & ALK TKI & 11 March 2016 [222] \\
\hline & & Entrectinib & Selective TKI & 15 August 2019 [16] \\
\hline \multirow{2}{*}{$\begin{array}{l}\text { MET exon } 14 \text { skipping } \\
\text { mutations }\end{array}$} & \multirow{2}{*}{ Metastatic } & Capmatinib & \multirow{2}{*}{ MET inhibitor } & 6 May 2020 [223] \\
\hline & & Tepotinib & & 3 February 2021 [224] \\
\hline
\end{tabular}




\subsection{EGFR}

Epidermal growth factor receptor (EGFR) is mutated in approximately $10 \%$ of Caucasian and potentially up to $50 \%$ of Asian NSCLC patients with limited or no smoking history [225]. The majority of EGFR mutations sensitive to targeted therapy lie within the tyrosine kinase domain, with exon 19 deletions or exon 21's L858R comprising the vast majority. Other rare lesions may also be sensitive to EGFR TKIs and include L861Q, G719X and S768I [226]. Current oral TKIs with FDA approved to treat advanced NSCLC patients whose tumors harbor sensitizing EGFR mutations include gefitinib, erlotinib (with or without ramucirumab), afatinib, dacomitinib and osimertinib.

Initially, first-generation oral TKIs gefitinib and erlotinib showed promising activity for inhibition of sensitizing EGFR mutations. In the randomized phase 3 trial, IPASS, firstline gefitinib was affiliated with an improved response rate versus platinum-based doublet chemotherapy ( $71 \%$ vs. $43 \%$ ) in Asian patients with EGFR mutations [227]. While this agent also showed a prolonged PFS, subsequent results did not translate into an improved OS, likely due to ensuing TKI use of the chemotherapy arm upon progression [228]. These findings were also confirmed in Caucasian NSCLC patients with EGFR mutations [229]. Likewise, EURTAC detailed a prolonged PFS for first-line erlotinib versus chemotherapy in European EGFR-mutated NSCLC patients (9.7 vs. 5.2 months; HR 0.37) [230]. FDA approval was also granted in 2020 to the combination of ramucirumab, a recombinant monoclonal antibody targeting vascular endothelial growth factor (VEGF) receptors, plus erlotinib for first-line use in metastatic NSCLC patients with exon 19 deletions or L858R mutations. Approval was granted based on results of a randomized phase 3 trial, RELAY, which showed a prolonged PFS for the combination versus erlotinib monotherapy (19.4 vs. 12.4 months; HR 0.59); a similar response rate of both arms was observed (approximately $75 \%$ ) [231]. However, $72 \%$ of patients in the combination arm had grade 3-4 adverse events including hypertension and transaminase abnormalities; one treatment-related death in the combination arm occurred. While FDA approved and an option to be discussed with patients, the niche of combinatorial strategies of EGFR TKIs with VEGF receptor inhibitors (e.g., ramucirumab or bevacizumab) [232] in the frontline setting warrants further exploration, especially when next-generation TKIs (see osimertinib below) are very efficacious and have a favorable toxicity profile.

Second-generation TKIs include afatinib and dacomitinib, which irreversibly inhibit multiple ErbB/HER receptors, including EGFR. Phase 3 LUX-Lung 3 showed an improvement of PFS in advanced lung adenocarcinoma patients with sensitizing EGFR mutations treated with afatinib compared to cisplatin plus pemetrexed (11.1 vs. 6.9 months). Additionally, afatinib was FDA approved in 2018 for uncommon mutations S768I, L861Q and/or G719X based upon combined analysis from the LUX-Lung 2, 3 and 6 trials [233,234]. Dacomitinib received FDA approval in 2018 following published results from the randomized phase 3 ARCHER1050 study, with updated data showing a prolongation of OS versus gefitinib in the first-line setting (median 34.1 vs. 26.8 months; HR 0.76) [235,236].

Initially FDA approved in 2017 following progression on another TKI based on its efficacy in the AURA3 trial against EGFR resistance mutation, T790M [237], third-generation TKI osimertinib is now considered a standard-of-care for untreated, advanced NSCLC patients with sensitizing EGFR aberrations based on the FLAURA study [238]. Most recently, secondary endpoint OS analysis was published, showing a significant prolongation of median OS in the osimertinib arm compared to TKIs gefitinib or erlotinib (38.6 vs. 31.8 months; HR 0.80) [239]. The attraction of osimertinib includes not only its activity against T790M, but a relatively mild toxicity profile with QT prolongation $(10 \%$, reduced cardiac ejection fraction (5\%), pneumonitis $(2 \%)$ and interstitial lung disease $(2 \%)$ with no treatment-related deaths. Further, osimertinib can induce durable intracranial responses, including in patients with leptomeningeal disease [240,241].

The clinical utility of osimertinib in EGFR-mutated NSCLC is extending to other indications. For example, the agent could be considered as a possible alternative to afatinib for advanced NSCLC patients whose tumor harbors uncommon EGFR mutations [242,243]. 
Additionally, the recently-published ADAURA trial [244] showed an impressive diseasefree survival benefit at two years with the addition of osimertinib to provider-determined adjuvant therapy in stage II and IIIA patients (90\% versus $44 \%$ for placebo; HR 0.17 ). Further, only $1 \%$ of patients receiving osimertinib developed CNS recurrence versus $10 \%$ of the placebo arm at two years. The adjuvant use of osimertinib was FDA approved on 18 December 2020 and extended to stage IB-IIIA NSCLC (non-squamous) patients whose tumors harbored EGFR exon 19 deletions or exon 21 L858R mutations [215]. This approval represents a paradigm shift, as increasing numbers of non-metastatic NSCLC patients will now receive molecular profiling whereas testing was previously relegated to the advanced setting. The additional genomic information received and potential uncovering of targetable molecular targets in the early-stage space provides a clinical challenge and opportunity for further study. Upon resistance to osimertinib, it is important to re-biopsy, if possible, and obtain tissue or liquid molecular profiling both to assess for small cell transformation, as well as potential targetable resistance mechanisms including MET amplifications, additional EGFR mutations or rare fusion events [245].

Finally, it should be noted that exon 20 insertion mutations represent the third most common alteration of EGFR and are generally not sensitive to TKIs. At this time, two agents selective to exon 20 insertions have received FDA Breakthrough Therapy Designation, amivantamab-vmjw [246] and mobocertinib [247]. On May 21, 2021, amivantamab, a bispecific antibody to EGFR and MET, was FDA approved for advanced NSCLC patients with EGFR exon 20 mutations after progression with chemotherapy. The approval was based upon the phase 1 CHRYSALIS study, which showed a $40 \%$ ORR with a median duration exceeding 11 months in 81 evaluated patients [246].

\subsection{ALK, ROS1}

Anaplastic lymphoma kinase (ALK; $2-5 \%$ of NSCLC) and ROS1 (1-3\%) rearrangements represent another subset of oncogenic drivers in NSCLC for which there are multiple effective targeted agents. Three precision drugs target both ALK and ROS1 (crizotinib (which also has activity against MET) [248-252], ceritinib [253] and lorlatinib) [254,255]. ALK can also be inhibited by alectinib (which also has activity against RET) [256-258] and brigatinib [259-262], while ROS1 is also inhibited by entrectinib (previously discussed for NTRK fusions) [263]. In the first-line setting for ALK-rearranged disease, current preference should be given to alectinib, brigatinib or lorlatinib over crizotinib, as all three agents showed improved efficacy in randomized phase 3 trials compared to the first-generation ALK inhibitor [255-258,261,262]. For example, updated data from the ALEX trial recently established an advantage of alectinib over crizotinib for mature PFS (median 34.8 vs. 10.9 months; HR 0.43) and median OS (not reached vs. 57.4 months; HR 0.67), which was also seen for patients with brain metastases [258]. While ceritinib remains an FDA-approved frontline option based on the ASCEND-4 trial [253], a direct comparison to crizotinib or another TKI has not been published to date.

The toxicity profile should be considered before the use of any TKI in the management of $A L K$-rearranged NSCLC, such as myalgia, edema, hepatotoxicity, interstitial lung disease/pneumonitis and bradycardia with alectinib and respiratory symptoms [264,265], vision change, amylase and lipase elevation, hypertension and similarly bradycardia with brigatinib. Although associated with undesirable toxicities including cognitive effects, mood changes, peripheral neuropathy and elevated triglycerides or cholesterol, lorlatinib is a third-generation TKI that has ample CNS penetration and has emerged as an effective agent with not only front-line activity, but efficacy at progression for multiple $A L K$ resistance mutations from early-generation inhibitors [255,266-268]. As with EGFR, the mechanism of resistance to $A L K$-inhibition should be sought with repeat tissue or liquid biopsy as targetable resistance mechanisms aside from $A L K$ mutations may be identified [269].

Of the three available agents that target ROS1, entrectinib and crizotinib are FDA approved in advanced ROS1-rearranged NSCLC and should be prioritized over ceritinib 
in the first-line setting. Further, in addition to pulmonary toxicity seen with almost all TKIs utilized in NSCLC, ceritinib use includes heightened gastrointestinal toxicities such as diarrhea, nausea and vomiting, hepatotoxicity and pancreatitis that may make it less tolerable than other agents [253]. Both entrectinib and crizotinib provide ORR of approximately $70-80 \%$, a significant portion of which are durable [252,263]; further, intracranial response of entrectinib is reported as $55 \%$.

Upon progression, lorlatinib has likewise emerged as a preferred agent subsequent to either crizotinib or entrectinib [270,271]. Next-generation TKIs such as repotrectinib, which has impressive CNS penetration and activity against ROS1, ALK and NTRK, are currently being evaluated for first-line or subsequent-line use [272].

\subsection{MET}

Alterations in oncogenic driver MET occur in at least 3-5\% of NSCLC and classically are affiliated with poor prognosis. While not all mutations are susceptible to targeted therapy, tumors with MET exon 14 lesions or a significantly elevated gene copy number may predict efficacy to TKIs [273]. In particular, MET exon 14 alterations are sensitive to inhibition with multi-kinase inhibitors crizotinib [274], cabozantinib [275], as well as recently FDA-approved selective inhibitors capmatinib [276,277] and tepotinib [277]. The GEOMETRY mono-1 trial reported a $68 \%$ response rate with capmatinib for untreated patients whose NSCLC contained MET exon 14 skipping mutations with a 12.6-month median duration of response. The authors reported a $40 \%$ response rate first-line for those harboring MET amplifications with a gene copy number of at least 10 [278]. Toxicity from this next-generation TKI is relatively modest and most frequently includes peripheral edema and nausea. Likewise, the efficacy of tepotinib was evaluated in the VISION trial, where 152 advanced NSCLC patients with exon 14 skipping mutations showed a $43 \%$ ORR (the same ORR was seen for treatment-naïve or those previously treated), with a median response duration of approximately 11 months. Combined with reports of effective CNS activity, capmatinib [278] or tepotinib [279,280] should be considered as the first-line options utilized for MET-directed therapy.

In addition to FDA-approved agents targeting sensitive alterations in EGFR, ALK, ROS1, BRAF, MET, RET and NTRK, the precision therapeutic arsenal in NSCLC may soon expand to other oncogenic drivers, including previously-cited KRAS G12C (13\% of all NSCLC) [196] and HER2 exon 20 mutations [146] lending further credence to the necessity of molecular profiling in this target-rich disease.

\section{Conclusions}

As we have attempted to show, advances in molecular profiling have enabled genomic classification of a patient's tumor, leading to the development, approval and availability of precision therapies including TKIs, ADCs and ICIs. With this ever-expanding arsenal of treatment options and increasing availability of next-generation sequencing, the utilization of molecular profiling is primed to expand to most advanced solid tumors into earlystage disease and include combinatorial precision regimens based on complex molecular findings [281]. It is incumbent for the modern oncologist to be well-versed regarding the range of potentially targetable aberrations available, be comfortable with a molecular profiling platform he or she trusts and be able to effectively interpret resultant data to help patients make informed decisions with regards to treatment.

Author Contributions: Conceptualization, M.K.S. and A.V.; methodology, not applicable; software, not applicable; validation, not applicable; formal analysis, not applicable; investigation, M.K.S., O.O., K.P. and A.V.; resources, not applicable; data curation, M.K.S., O.O., K.P. and A.V.; writing-original draft preparation, M.K.S., O.O., K.P. and A.V.; writing-review and editing, M.K.S., O.O., K.P. and A.V.; visualization, A.V.; supervision, A.V.; project administration, A.V.; funding acquisition, not applicable. All authors have read and agreed to the published version of the manuscript.

Funding: This research received no external funding. 
Data Availability Statement: All data reported in this paper is available in the cited references, available either at pubmed.gov or at the websites within the citations.

Conflicts of Interest: M.S.K., O.O. and K.P. declare no conflict of interest. A.V. has received research support from Amgen, is a consultant for Bristol-Myers Squibb and Elsevier, and participates in advisory boards for Roche/Genentech, Mirati, and Bristol-Myers Squibb.

\section{References}

1. Schwartzberg, L.; Kim, E.S.; Liu, D.; Schrag, D. Precision Oncology: Who, How, What, When, and When Not? Am. Soc. Clin. Oncol. Educ. Book 2017, 37, 160-169. [CrossRef] [PubMed]

2. Venter, J.C.; Adams, M.D.; Myers, E.W.; Li, P.W.; Mural, R.J.; Sutton, G.G.; Smith, H.O.; Yandell, M.; Evans, C.A.; Holt, R.A.; et al. The sequence of the human genome. Science 2001, 291, 1304-1351. [CrossRef]

3. Tao, J.J.; Schram, A.M.; Hyman, D.M. Basket Studies: Redefining Clinical Trials in the Era of Genome-Driven Oncology. Annu. Rev. Med. 2018, 69, 319-331. [CrossRef] [PubMed]

4. Verma, M. Personalized medicine and cancer. J. Pers Med. 2012, 2, 1-14. [CrossRef]

5. Druker, B.J.; Talpaz, M.; Resta, D.J.; Peng, B.; Buchdunger, E.; Ford, J.M.; Lydon, N.B.; Kantarjian, H.; Capdeville, R.; Ohno-Jones, S.; et al. Efficacy and safety of a specific inhibitor of the BCR-ABL tyrosine kinase in chronic myeloid leukemia. N. Engl. J. Med. 2001, 344, 1031-1037. [CrossRef] [PubMed]

6. Vogel, C.L.; Cobleigh, M.A.; Tripathy, D.; Gutheil, J.C.; Harris, L.N.; Fehrenbacher, L.; Slamon, D.J.; Murphy, M.; Novotny, W.F.; Burchmore, M.; et al. Efficacy and safety of trastuzumab as a single agent in first-line treatment of HER2-overexpressing metastatic breast cancer. J. Clin. Oncol. 2002, 20, 719-726. [CrossRef]

7. Slamon, D.J.; Leyland-Jones, B.; Shak, S.; Fuchs, H.; Paton, V.; Bajamonde, A.; Fleming, T.; Eiermann, W.; Wolter, J.; Pegram, M.; et al. Use of chemotherapy plus a monoclonal antibody against HER2 for metastatic breast cancer that overexpresses HER2. N. Engl. J. Med. 2001, 344, 783-792. [CrossRef]

8. VanderWalde, A.; Grothey, A.; Vaena, D.; Vidal, G.; ElNaggar, A.; Bufalino, G.; Schwartzberg, L. Establishment of a Molecular Tumor Board (MTB) and Uptake of Recommendations in a Community Setting. J. Pers. Med. 2020, 10, 252. [CrossRef]

9. FDA Grants Accelerated Approval to Pembrolizumab for First Tissue/Site Agnostic Indication. Available online: https: / / www.fda.gov / drugs/resources-information-approved-drugs/fda-grants-accelerated-approval-pembrolizumabfirst-tissuesite-agnostic-indication (accessed on 21 April 2021).

10. FDA Grants Nivolumab Accelerated Approval for MSI-H or dMMR Colorectal Cancer. Content Current as of: 08/01/2017. Available online: https://www.fda.gov/drugs/resources-information-approved-drugs/fda-grants-nivolumab-acceleratedapproval-msi-h-or-dmmr-colorectal-cancer (accessed on 28 April 2021).

11. FDA Grants Accelerated Approval to Ipilimumab for MSI-H or dMMR Metastatic Colorectal Cancer. Content Current as of: 07/11/2018. Available online: https://www.fda.gov/drugs/resources-information-approved-drugs/fda-grants-acceleratedapproval-ipilimumab-msi-h-or-dmmr-metastatic-colorectal-cancer (accessed on 28 April 2021).

12. FDA Approves Pembrolizumab for First-Line Treatment of MSI-H/dMMR Colorectal Cancer. Content Current as of: 06/30/2020. Available online: https://www.fda.gov/drugs/drug-approvals-and-databases/fda-approves-pembrolizumab-first-linetreatment-msi-hdmmr-colorectal-cancer (accessed on 28 April 2021).

13. FDA News Release. FDA Approves Immunotherapy for Endometrial Cancer with Specific Biomarker. For Immediate Release: April 22, 2021. Available online: https:/ / www.fda.gov/news-events/press-announcements/fda-approves-immunotherapyendometrial-cancer-specific-biomarker (accessed on 24 April 2021).

14. FDA Approves Pembrolizumab for Adults and Children with TMB-H Solid Tumors. Available online: https://www.fda.gov/ drugs/drug-approvals-and-databases/fda-approves-pembrolizumab-adults-and-children-tmb-h-solid-tumors (accessed on 22 April 2021).

15. FDA Approves Larotrectinib for Solid Tumors with NTRK Gene Fusions. Available online: https://www.fda.gov/drugs / fdaapproves-larotrectinib-solid-tumors-ntrk-gene-fusions (accessed on 21 April 2021).

16. FDA Approves Entrectinib for NTRK Solid Tumors and ROS-1 NSCLC. Available online: https://www.fda.gov/drugs / resourcesinformation-approved-drugs/fda-approves-entrectinib-ntrk-solid-tumors-and-ros-1-nsclc (accessed on 21 April 2021).

17. Kim, G.; McKee, A.E.; Ning, Y.M.; Hazarika, M.; Theoret, M.; Johnson, J.R.; Xu, Q.C.; Tang, S.; Sridhara, R.; Jiang, X.; et al. FDA approval summary: Vemurafenib for treatment of unresectable or metastatic melanoma with the BRAFV600E mutation. Clin. Cancer Res. 2014, 20, 4994-5000. [CrossRef]

18. Thompson, C.A. FDA approves two new drugs against advanced melanoma. Am. J. Health Syst. Pharm. 2013, 70, 1094. [CrossRef]

19. Novel Drug Approvals for 2015. Content Current as of: 11/15/2019. Available online: https://www.fda.gov/drugs/newdrugs-fda-cders-new-molecular-entities-and-new-therapeutic-biological-products/novel-drug-approvals-2015 (accessed on 29 April 2021).

20. Combination therapy approved for melanoma. Cancer Discov. 2014, 4, 262

21. FDA Approves Encorafenib and Binimetinib in Combination for Unresectable or Metastatic Melanoma with BRAF Mutations. Content Current as of: 06/27/18. Available online: https://www.fda.gov/drugs/resources-information-approved-drugs/fdaapproves-encorafenib-and-binimetinib-combination-unresectable-or-metastatic-melanoma-braf (accessed on 28 April 2021). 
22. FDA Approves Dabrafenib Plus Trametinib for Adjuvant Treatment of Melanoma with BRAF V600E or V600K Mutations. Content Current as of: 05/01/2018. Available online: https:/ /www.fda.gov/drugs/resources-information-approved-drugs/ fda-approves-dabrafenib-plus-trametinib-adjuvant-treatment-melanoma-braf-v600e-or-v600k-mutations (accessed on 28 April 2021).

23. FDA Approves Atezolizumab for BRAF V600 Unresectable or Metastatic Melanoma. Content Current as of: 07/31/2020. Available online: https:/ / www.fda.gov/drugs/resources-information-approved-drugs/fda-approves-atezolizumab-braf-v6 00-unresectable-or-metastatic-melanoma (accessed on 28 April 2021).

24. Odogwu, L.; Mathieu, L.; Blumenthal, G.; Larkins, E.; Goldberg, K.B.; Griffin, N.; Bijwaard, K.; Lee, E.Y.; Philip, R.; Jiang, X.; et al. FDA Approval Summary: Dabrafenib and Trametinib for the Treatment of Metastatic Non-Small Cell Lung Cancers Harboring BRAF V600E Mutations. Oncologist 2018, 23, 740-745. [CrossRef]

25. FDA NEWS RELEASE: FDA Approves New Uses for Two Drugs Administered Together for the Treatment of BRAF-Positive Anaplastic Thyroid Cancer. Content Current as of: 05/04/2018. Available online: https://www.fda.gov/news-events/ press-announcements / fda-approves-new-uses-two-drugs-administered-together-treatment-braf-positive-anaplastic-thyroid (accessed on 28 April 2021).

26. FDA Approves Encorafenib in Combination with Cetuximab for Metastatic Colorectal Cancer with a BRAF V600E Mutation. Content Current as of: 04/09/2020. Available online: https://www.fda.gov/drugs/resources-information-approved-drugs/fdaapproves-encorafenib-combination-cetuximab-metastatic-colorectal-cancer-braf-v600e-mutation (accessed on 28 April 2021).

27. Monoclonal antibody approved for metastatic breast cancer. Oncology 1998, 12, 1727.

28. Blumenthal, G.M.; Scher, N.S.; Cortazar, P.; Chattopadhyay, S.; Tang, S.; Song, P.; Li, Q.; Ringgold, K.; Pilaro, A.M.; Tilley, A.; et al. First FDA approval of dual anti-HER2 regimen: Pertuzumab in combination with trastuzumab and docetaxel for HER2-positive metastatic breast cancer. Clin. Cancer Res. 2013, 19, 4911-4916. [CrossRef]

29. Amiri-Kordestani, L.; Blumenthal, G.M.; Xu, Q.C.; Zhang, L.; Tang, S.W.; Ha, L.; Weinberg, W.C.; Chi, B.; Candau-Chacon, R.; Hughes, P.; et al. FDA approval: Ado-trastuzumab emtansine for the treatment of patients with HER2-positive metastatic breast cancer. Clin. Cancer Res. 2014, 20, 4436-4441. [CrossRef]

30. Singh, H.; Walker, A.J.; Amiri-Kordestani, L.; Cheng, J.; Tang, S.; Balcazar, P.; Barnett-Ringgold, K.; Palmby, T.R.; Cao, X.; Zehng, N.; et al. U.S. Food and Drug Administration Approval: Neratinib for the Extended Adjuvant Treatment of Early-Stage HER2-Positive Breast Cancer. Clin. Cancer Res. 2018, 24, 3486-3491. [CrossRef] [PubMed]

31. FDA Approves Fam-Trastuzumab Deruxtecan-Nxki for Unresectable or Metastatic HER2-Positive Breast Cancer. Content Current as of: 12/20/2019. Available online: https://www.fda.gov/drugs/resources-information-approved-drugs/fda-approves-famtrastuzumab-deruxtecan-nxki-unresectable-or-metastatic-her2-positive-breast-cancer (accessed on 26 April 2021).

32. Ryan, Q.; Ibrahim, A.; Cohen, M.H.; Johnson, J.; Ko, C.W.; Sridhara, R.; Justice, R.; Pazdur, R. FDA drug approval summary: Lapatinib in combination with capecitabine for previously treated metastatic breast cancer that overexpresses HER-2. Oncologist 2008, 13, 1114-1119. [CrossRef] [PubMed]

33. FDA Approves Tucatinib for Patients with HER2-Positive Metastatic Breast Cancer. Content Current as of: 04/20/2020. Available online: https: / / www.fda.gov/drugs/resources-information-approved-drugs/fda-approves-tucatinib-patients-her2-positivemetastatic-breast-cancer (accessed on 26 April 2021).

34. 2010 Notifications. Content Current as of: 02/13/2018. Available online: https://www.fda.gov/drugs/resources-informationapproved-drugs/2010-notifications (accessed on 27 April 2021).

35. FDA Approves Fam-Trastuzumab Deruxtecan-Nxki for HER2-Positive Gastric Adenocarcinomas. Content Current as of: 01/15/2021. Available online: https:/ / www.fda.gov/drugs/drug-approvals-and-databases/fda-approves-fam-trastuzumabderuxtecan-nxki-her2-positive-gastric-adenocarcinomas (accessed on 27 April 2021).

36. FDA Approves Selpercatinib for Lung and Thyroid Cancers with RET Gene Mutations or Fusions. Content Current as of 05/11/2020. Available online: https:/ / www.fda.gov/drugs/drug-approvals-and-databases/fda-approves-selpercatinib-lungand-thyroid-cancers-ret-gene-mutations-or-fusions (accessed on 26 April 2021).

37. FDA Approves Pralsetinib for Lung Cancer with RET Gene Fusions. Available online: https://www.fda.gov/drugs/resourcesinformation-approved-drugs/fda-approves-pralsetinib-lung-cancer-ret-gene-fusions (accessed on 22 April 2021).

38. Arora, S.; Balasubramaniam, S.; Zhang, H.; Berman, T.; Narayan, P.; Suzman, D.; Bloomquist, E.; Tang, S.; Gong, Y.; Sridhara, R.; et al. FDA Approval Summary: Olaparib Monotherapy or in Combination with Bevacizumab for the Maintenance Treatment of Patients with Advanced Ovarian Cancer. Oncologist 2021, 26, e164-e172. [CrossRef]

39. Balasubramaniam, S.; Beaver, J.A.; Horton, S.; Fernandes, L.L.; Tang, S.; Horne, H.N.; Liu, J.; Liu, C.; Schrieber, S.J.; Yu, J.; et al. FDA Approval Summary: Rucaparib for the Treatment of Patients with Deleterious BRCA Mutation-Associated Advanced Ovarian Cancer. Clin. Cancer Res. 2017, 23, 7165-7170. [CrossRef] [PubMed]

40. FDA Approves Rucaparib for Maintenance Treatment of Recurrent Ovarian, Fallopian Tube, or Primary Peritoneal Cancer. Content Current as of: 04/06/2018. Available online: https:/ /www.fda.gov/drugs/resources-information-approved-drugs /fdaapproves-rucaparib-maintenance-treatment-recurrent-ovarian-fallopian-tube-or-primary-peritoneal (accessed on 27 April 2021).

41. Ison, G.; Howie, L.J.; Amiri-Kordestani, L.; Zhang, L.; Tang, S.; Sridhara, R.; Pierre, V.; Charlab, R.; Ramamoorthy, A.; Song, P.; et al. FDA Approval Summary: Niraparib for the Maintenance Treatment of Patients with Recurrent Ovarian Cancer in Response to Platinum-Based Chemotherapy. Clin. Cancer Res. 2018, 24, 4066-4071. [CrossRef] 
42. FDA Approves Niraparib for HRD-Positive Advanced Ovarian Cancer. Content Current as of: 10/23/2019. Available online: https: / / www.fda.gov/drugs/resources-information-approved-drugs/fda-approves-niraparib-hrd-positive-advancedovarian-cancer (accessed on 27 April 2021).

43. FDA Approves Olaparib for Germline BRCA-Mutated Metastatic Breast Cancer. Content Current as of: 01/12/2018. Available online: https: / / www.fda.gov / drugs/resources-information-approved-drugs/fda-approves-olaparib-germline-brca-mutatedmetastatic-breast-cancer (accessed on 27 April 2021).

44. FDA Approves Talazoparib for gBRCAm HER2-Negative Locally Advanced or Metastatic Breast Cancer. Content Current as of: 12/14/2018. Available online: https:/ / www.fda.gov/drugs/drug-approvals-and-databases/fda-approves-talazoparib-gbrcamher2-negative-locally-advanced-or-metastatic-breast-cancer (accessed on 27 April 2021).

45. FDA Approves Olaparib for gBRCAm Metastatic Pancreatic Adenocarcinoma. Content Current as of: 12/30/2019. Available online: https:/ / www.fda.gov/drugs/resources-information-approved-drugs/fda-approves-olaparib-gbrcam-metastaticpancreatic-adenocarcinoma (accessed on 27 April 2021).

46. Anscher, M.S.; Chang, E.; Gao, X.; Gong, Y.; Weinstock, C.; Bloomquist, E.; Adeniyi, O.; Charlab, R.; Zimmerman, S.; SerlemitsosDay, M.; et al. FDA Approval Summary: Rucaparib for the Treatment of Patients with Deleterious BRCA-Mutated Metastatic Castrate-Resistant Prostate Cancer. Oncologist 2021, 26, 139-146. [CrossRef]

47. FDA Approves Olaparib for HRR Gene-Mutated Metastatic Castration-Resistant Prostate Cancer. Content Current as of: 05/20/2020. Available online: https://www.fda.gov/drugs/drug-approvals-and-databases/fda-approves-olaparib-hrr-genemutated-metastatic-castration-resistant-prostate-cancer (accessed on 27 April 2021).

48. Marcus, L.; Lemery, S.J.; Keegan, P.; Pazdur, R. FDA Approval Summary: Pembrolizumab for the Treatment of Microsatellite Instability-High Solid Tumors. Clin. Cancer Res. 2019, 25, 3753-3758. [CrossRef]

49. Le, D.T.; Uram, J.N.; Wang, H.; Bartlett, B.R.; Kemberling, H.; Eyring, A.D.; Slora, A.D.; Luber, B.S.; Azad, N.S.; Laheru, D.; et al. PD-1 Blockade in Tumors with Mismatch-Repair Deficiency. N. Engl. J. Med. 2015, 372, 2509-2520. [CrossRef] [PubMed]

50. Le, D.T.; Kim, T.W.; van Cutsem, E.; Geva, R.; Jäger, D.; Hara, H.; Burge, M.; O’Neil, B.; Kavan, P.; Yoshino, T.; et al. Phase II Open-Label Study of Pembrolizumab in Treatment-Refractory, Microsatellite Instability-High/Mismatch Repair-Deficient Metastatic Colorectal Cancer: KEYNOTE-164. J. Clin. Oncol. 2020, 38, 11-19. [CrossRef] [PubMed]

51. Marabelle, A.; Le, D.T.; Ascierto, P.A.; Di Giacomo, A.M.; de Jesus-Acosta, A.; Delord, J.P.; Geva, R.; Gottfried, M.; Penel, N.; Hansen, A.R.; et al. Efficacy of Pembrolizumab in Patients with Noncolorectal High Microsatellite Instability/Mismatch Repair-Deficient Cancer: Results From the Phase II KEYNOTE-158 Study. J. Clin. Oncol. 2020, 38, 1-10. [CrossRef] [PubMed]

52. Overman, M.J.; McDermott, R.; Leach, J.L.; Lonardi, S.; Lenz, H.J.; Morse, M.A.; Desai, J.; Hill, A.; Axelson, M.; Moss, R.A.; et al. Nivolumab in patients with metastatic DNA mismatch repair-deficient or microsatellite instability-high colorectal cancer (CheckMate 142): An open-label, multicentre, phase 2 study. Lancet Oncol. 2017, 18, 1182-1191. [CrossRef]

53. Overman, M.J.; Lonardi, S.; Wong, K.Y.M.; Lenz, H.J.; Gelsomino, F.; Aglietta, M.; Morse, M.A.; Van Cutsem, E.; McDermott, R.; Hill, A.; et al. Durable Clinical Benefit with Nivolumab Plus Ipilimumab in DNA Mismatch Repair-Deficient/Microsatellite Instability-High Metastatic Colorectal Cancer. J. Clin. Oncol. 2018, 36, 773-779. [CrossRef]

54. André, T.; Shiu, K.K.; Kim, T.W.; Jensen, B.V.; Jensen, L.H.; Punt, C.; Smith, D.; Garcia-Carbonero, R.; Benavides, M.; Gibbs, P.; et al. Pembrolizumab in Microsatellite-Instability-High Advanced Colorectal Cancer. N. Engl. J. Med. 2020, 383, 2207-2218. [CrossRef]

55. Mandal, R.; Samstein, R.M.; Lee, K.W.; Havel, J.J.; Wang, H.; Krishna, C.; Sabio, E.Y.; Makarov, V.; Kuo, F.; Blecua, P.; et al. Genetic diversity of tumors with mismatch repair deficiency influences anti-PD-1 immunotherapy response. Science 2019, 364, 485-491. [CrossRef]

56. Sidaway, P. MSI-H: A truly agnostic biomarker? Nat. Rev. Clin. Oncol. 2020, 17, 68. [CrossRef]

57. Rosen, E.Y.; Goldman, D.A.; Hechtman, J.F.; Benayed, R.; Schram, A.M.; Cocco, E.; Shifman, S.; Gong, Y.; Kundra, R.; Solomon, J.p.; et al. TRK Fusions Are Enriched in Cancers with Uncommon Histologies and the Absence of Canonical Driver Mutations. Clin. Cancer Res. 2020, 26, 1624-1632. [CrossRef] [PubMed]

58. Drilon, A.; Laetsch, T.W.; Kummar, S.; DuBois, S.G.; Lassen, U.N.; Demetri, G.D.; Nathenson, M.; Doebele, R.C.; Farago, A.F.; Pappo, A.S.; et al. Efficacy of Larotrectinib in TRK Fusion-Positive Cancers in Adults and Children. N. Engl. J. Med. 2018, 378, 731-739. [CrossRef]

59. Hong, D.S.; DuBois, S.G.; Kummar, S.; Farago, A.F.; Albert, C.M.; Rohrberg, K.S.; van Tilburg, C.M.; Nagasubramanian, R.; Berlin, J.D.; Federman, N.; et al. Larotrectinib in patients with TRK fusion-positive solid tumours: A pooled analysis of three phase $1 / 2$ clinical trials. Lancet Oncol. 2020, 21, 531-540. [CrossRef]

60. Doebele, R.C.; Drilon, A.; Paz-Ares, L.; Siena, S.; Shaw, A.T.; Farago, A.F.; Blakely, C.M.; Seto, T.; Cho, B.C.; Tosi, D.; et al. Entrectinib in patients with advanced or metastatic NTRK fusion-positive solid tumours: Integrated analysis of three phase 1-2 trials. Lancet Oncol. 2020, 21, 271-282. [CrossRef]

61. Drilon, A. TRK inhibitors in TRK fusion-positive cancers. Ann. Oncol. 2019, 30 (Suppl. 8), viii23-viii30. [CrossRef]

62. Drilon, A.; Ou, S.I.; Cho, B.C.; Kim, D.W.; Lee, J.; Lin, J.J.; Zhu, V.W.; Ahn, M.J.; Camidge, D.R.; Nguyen, J.; et al. Repotrectinib (TPX-0005) Is a Next-Generation ROS1/TRK/ALK Inhibitor That Potently Inhibits ROS1/TRK/ALK Solvent-Front Mutations. Cancer Discov. 2018, 8, 1227-1236. [CrossRef]

63. Drilon, A.; Zhai, D.; Deng, W.; Zhang, J.; Lee, D.; Rogers, E.; Whitten, J.; Huang, Z.; Graber, A.; Liu, J.; et al. Abstract 442: Repotrectinib, a next generation TRK inhibitor, overcomes TRK resistance mutations including solvent front, gatekeeper and compound mutations. Cancer Res. 2019, 79 (Suppl. 13), 442. 
64. Drilon, A.; Nagasubramanian, R.; Blake, J.F.; Ku, N.; Touch, B.B.; Ebata, K.; Smith, S.; Lauriault, V.; Kolakowsky, G.R.; Brandhuber, B.J.; et al. A Next-Generation TRK Kinase Inhibitor Overcomes Acquired Resistance to Prior TRK Kinase Inhibition in Patients with TRK Fusion-Positive Solid Tumors. Cancer Discov. 2017, 7, 963-972. [CrossRef]

65. Marabelle, A.; Fakih, M.; Lopez, J.; Shah, M.; Shapira-Frommer, R.; Nakagawa, K.; Chung, H.C.; Kindler, H.L.; Lopez-Martin, J.A.; Miller, J.H., Jr.; et al. Association of tumour mutational burden with outcomes in patients with advanced solid tumours treated with pembrolizumab: Prospective biomarker analysis of the multicohort, open-label, phase 2 KEYNOTE-158 study. Lancet Oncol. 2020, 21, 1353-1365. [CrossRef]

66. Prasad, V.; Addeo, A. The FDA approval of pembrolizumab for patients with TMB >10 mut/Mb: Was it a wise decision? No. Ann. Oncol. 2020, 31, 1112-1114. [CrossRef]

67. McGrail, D.J.; Pilié, P.G.; Rashid, N.U.; Voorwerk, L.; Slagter, M.; Kok, M.; Jonasch, E.; Khasraw, M.; Heimberger, A.B.; Lim, B.; et al. High tumor mutation burden fails to predict immune checkpoint blockade response across all cancer types. Ann. Oncol. 2021, 32, 661-672. [CrossRef]

68. Subbiah, V.; Solit, D.B.; Chan, T.A.; Kurzrock, R. The FDA approval of pembrolizumab for adult and pediatric patients with tumor mutational burden $(\mathrm{TMB}) \geq 10$ : A decision centered on empowering patients and their physicians. Ann. Oncol. 2020, 31, 1115-1118. [CrossRef]

69. Wang, F.; Zhao, Q.; Wang, Y.N.; Jin, Y.; He, M.M.; Liu, Z.X.; Xu, R.H. Evaluation of POLE and POLD1 Mutations as Biomarkers for Immunotherapy Outcomes Across Multiple Cancer Types. JAMA Oncol. 2019, 5, 1504-1506. [CrossRef]

70. Goodman, A.M.; Piccioni, D.; Kato, S.; Boichard, A.; Wang, H.Y.; Frampton, G.; Lippman, S.M.; Connelly, C.; Fabrizio, D.; Miller, V.; et al. Prevalence of PDL1 Amplification and Preliminary Response to Immune Checkpoint Blockade in Solid Tumors. JAMA Oncol. 2018, 4, 1237-1244. [CrossRef] [PubMed]

71. Straub, M.; Drecoll, E.; Pfarr, N.; Weichert, W.; Langer, R.; Hapfelmeier, A.; Gotz, C.; Wolff, K.D.; Kolk, A.; Specht, K. CD274/PD-L1 gene amplification and PD-L1 protein expression are common events in squamous cell carcinoma of the oral cavity. Oncotarget 2016, 7, 12024-12034. [CrossRef] [PubMed]

72. Sorscher, S.; Resnick, J.; Goodman, M. First Case Report of a Dramatic Radiographic Response to a Checkpoint Inhibitor in a Patient with Proficient Mismatch Repair Gene Expressing Metastatic Colorectal Cancer. JCO Precis. Oncol. 2017, 1, 1-4. [CrossRef]

73. Ikeda, S.; Goodman, A.M.; Cohen, P.R.; Jensen, T.J.; Ellison, C.K.; Frampton, G.; Miller, V.; Patel, S.P.; Kurzrock, R. Metastatic basal cell carcinoma with amplification of PD-L1: Exceptional response to anti-PD1 therapy. NPJ Genom. Med. 2016, 1, 16037. [CrossRef] [PubMed]

74. Flaherty, K.T.; Puzanov, I.; Kim, K.B.; Ribas, A.; McArthur, G.A.; Sosman, J.A.; O’Dwyer, P.J.; Lee, R.J.; Grippo, J.F.; Nolop, K.; et al. Inhibition of mutated, activated BRAF in metastatic melanoma. N. Engl. J. Med. 2010, 363, 809-819. [CrossRef]

75. Hauschild, A.; Grob, J.J.; Demidov, L.V.; Jouary, T.; Gutzmer, R.; Millward, M.; Rutkowski, P.; Blank, C.U.; Miller, W.H., Jr.; Kaempgen, E.; et al. Dabrafenib in BRAF-mutated metastatic melanoma: A multicentre, open-label, phase 3 randomised controlled trial. Lancet 2012, 380, 358-365. [CrossRef]

76. Flaherty, K.T.; Robert, C.; Hersey, P.; Nathan, P.; Garbe, C.; Milhem, M.; Demidov, L.V.; Hassel, J.C.; Rutkowski, P.; Mohr, P.; et al. Improved survival with MEK inhibition in BRAF-mutated melanoma. N. Engl. J. Med. 2012, 367, 107-114. [CrossRef] [PubMed]

77. Long, G.V.; Trefzer, U.; Davies, M.A.; Kefford, R.F.; Ascierto, P.A.; Chapman, P.B.; Puzanov, I.; Hauschild, A.; Robert, C.; Algazi, A.; et al. Dabrafenib in patients with Val600Glu or Val600Lys BRAF-mutant melanoma metastatic to the brain (BREAK-MB): A multicentre, open-label, phase 2 trial. Lancet Oncol. 2012, 13, 1087-1095. [CrossRef]

78. Bucheit, A.D.; Davies, M.A. Emerging insights into resistance to BRAF inhibitors in melanoma. Biochem. Pharmacol. 2014, 87, 381-389. [CrossRef] [PubMed]

79. Long, G.V.; Stroyakovskiy, D.; Gogas, H.; Levchenko, E.; de Braud, F.; Larkin, J.; Garbe, C.; Jouary, T.; Hauschild, A.; Grob, J.J.; et al. Combined BRAF and MEK inhibition versus BRAF inhibition alone in melanoma. N. Engl. J. Med. 2014, 371, 1877-1888. [CrossRef]

80. Long, G.V.; Stroyakovskiy, D.; Gogas, H.; Levchenko, E.; de Braud, F.; Larkin, J.; Garbe, C.; Jouary, T.; Hauschild, A.; Grob, J.J.; et al. Dabrafenib and trametinib versus dabrafenib and placebo for Val600 BRAF-mutant melanoma: A multicentre, double-blind, phase 3 randomised controlled trial. Lancet 2015, 386, 444-451. [CrossRef]

81. Long, G.V.; Flaherty, K.T.; Stroyakovskiy, D.; Gogas, H.; Levchenko, E.; de Braud, F.; Larkin, J.; Garbe, C.; Jouary, T.; Hauschild, A.; et al. Dabrafenib plus trametinib versus dabrafenib monotherapy in patients with metastatic BRAF V600E/K-mutant melanoma: Long-term survival and safety analysis of a phase 3 study. Ann. Oncol. 2017, 28, 1631-1639. [CrossRef]

82. Robert, C.; Karaszewska, B.; Schachter, J.; Rutkowski, P.; Mackiewicz, A.; Stroiakovski, D.; Lichinitser, M.; Dummer, R.; Grange, F.; Mortier, L.; et al. Improved overall survival in melanoma with combined dabrafenib and trametinib. N. Engl. J. Med. 2015, 372, 30-39. [CrossRef]

83. Robert, C.; Grob, J.J.; Stroyakovskiy, D.; Karaszewska, B.; Hauschild, A.; Levchenko, E.; Chiarion Sileni, V.; Schachter, J.; Garbe, C.; Bondarenko, I.; et al. Five-Year Outcomes with Dabrafenib plus Trametinib in Metastatic Melanoma. N. Engl. J. Med. 2019, 381, 626-636. [CrossRef]

84. Larkin, J.; Ascierto, P.A.; Dréno, B.; Atkinson, V.; Liszkay, G.; Maio, M.; Mandala, M.; Demidov, L.; Stroyakovskiy, D.; Thomas, L.; et al. Combined vemurafenib and cobimetinib in BRAF-mutated melanoma. N. Engl. J. Med. 2014, 371, 1867-1876. [CrossRef] 
85. Ascierto, P.A.; McArthur, G.A.; Dréno, B.; Atkinson, V.; Liszkay, G.; Di Giacomo, A.M.; Mandala, M.; Demidov, L.; Stroyakovskiy, D.; Thomas, L.; et al. Cobimetinib combined with vemurafenib in advanced BRAF(V600)-mutant melanoma (coBRIM): Updated efficacy results from a randomised, double-blind, phase 3 trial. Lancet Oncol. 2016, 17, 1248-1260. [CrossRef]

86. Dummer, R.; Ascierto, P.A.; Gogas, H.J.; Arance, A.; Mandala, M.; Liszkay, G.; Garbe, C.; Schadendorf, D.; Krajsova, I.; Gutzmer, R.; et al. Encorafenib plus binimetinib versus vemurafenib or encorafenib in patients with BRAF-mutant melanoma (COLUMBUS): A multicentre, open-label, randomised phase 3 trial. Lancet Oncol. 2018, 19, 603-615. [CrossRef]

87. Dummer, R.; Ascierto, P.A.; Gogas, H.J.; Arance, A.; Mandala, M.; Liszkay, G.; Garbe, C.; Schadendorf, D.; Krajsova, I.; Gutzmer, R.; et al. Overall survival in patients with BRAF-mutant melanoma receiving encorafenib plus binimetinib versus vemurafenib or encorafenib (COLUMBUS): A multicentre, open-label, randomised, phase 3 trial. Lancet Oncol. 2018, 19, 1315-1327. [CrossRef]

88. Ascierto, P.A.; Dummer, R.; Gogas, H.J.; Flaherty, K.T.; Arance, A.; Mandala, M.; Liszkay, G.; Garbe, C.; Schadendorf, D.; Krajsova, I.; et al. Update on tolerability and overall survival in COLUMBUS: Landmark analysis of a randomised phase 3 trial of encorafenib plus binimetinib vs vemurafenib or encorafenib in patients with BRAF V600-mutant melanoma. Eur. J. Cancer 2020, 126, 33-44. [CrossRef]

89. Gogas, H.J.; Flaherty, K.T.; Dummer, R.; Ascierto, P.A.; Arance, A.; Mandala, M.; Liszkay, G.; Garbe, C.; Schadendorf, D.; Krajsova, I.; et al. Adverse events associated with encorafenib plus binimetinib in the COLUMBUS study: Incidence, course and management. Eur. J. Cancer 2020, 119, 97-106. [CrossRef] [PubMed]

90. Long, G.V.; Hauschild, A.; Santinami, M.; Atkinson, V.; Mandalà, M.; Chiarion-Sileni, V.; Larkin, J.; Nyakas, M.; Dutriaux, C.; Haydon, A.; et al. Adjuvant Dabrafenib plus Trametinib in Stage III BRAF-Mutated Melanoma. N. Engl. J. Med. 2017, 377, 1813-1823. [CrossRef]

91. Dummer, R.; Hauschild, A.; Santinami, M.; Atkinson, V.; Mandalà, M.; Kirkwood, J.M.; Chiarion Sileni, V.; Larkin, J.; Nyakas, M.; Dutriaux, C.; et al. Five-Year Analysis of Adjuvant Dabrafenib plus Trametinib in Stage III Melanoma. N. Engl. J. Med. 2020, 383, 1139-1148. [CrossRef]

92. Weber, J.; Mandala, M.; Del Vecchio, M.; Gogas, H.J.; Arance, A.M.; Cowey, C.L.; Dalle, S.; Schenker, M.; Chiarion-Sileni, V.; Marquez-Rodas, I.; et al. Adjuvant Nivolumab versus Ipilimumab in Resected Stage III or IV Melanoma. N. Engl. J. Med. 2017, 377, 1824-1835. [CrossRef]

93. Eggermont, A.M.M.; Blank, C.U.; Mandala, M.; Long, G.V.; Atkinson, V.; Dalle, S.; Haydon, A.; Lichinitser, M.; Khattak, A.; Carlino, M.S.; et al. Adjuvant Pembrolizumab versus Placebo in Resected Stage III Melanoma. N. Engl. J. Med. 2018, 378, 1789-1801. [CrossRef]

94. Ribas, A.; Puzanov, I.; Dummer, R.; Schadendorf, D.; Hamid, O.; Robert, C.; Hodi, F.S.; Schachter, J.; Pavlick, A.C.; Lewis, K.D.; et al. Pembrolizumab versus investigator-choice chemotherapy for ipilimumab-refractory melanoma (KEYNOTE-002): A randomised, controlled, phase 2 trial. Lancet Oncol. 2015, 16, 908-918. [CrossRef]

95. Robert, C.; Schachter, J.; Long, G.V.; Arance, A.; Grob, J.J.; Mortier, L.; Daud, A.; Carlino, M.S.; McNeil, C.; Lotem, M.; et al. Pembrolizumab versus Ipilimumab in Advanced Melanoma. N. Engl. J. Med. 2015, 372, 2521-2532. [CrossRef] [PubMed]

96. Larkin, J.; Chiarion-Sileni, V.; Gonzalez, R.; Grob, J.J.; Cowey, C.L.; Lao, C.D.; Schadendorf, D.; Dummer, R.; Smylie, M.; Rutkowski, P.; et al. Combined Nivolumab and Ipilimumab or Monotherapy in Untreated Melanoma. N. Engl. J. Med. 2015, 373, 23-34. [CrossRef]

97. Weber, J.S.; D’Angelo, S.P.; Minor, D.; Hodi, F.S.; Gutzmer, R.; Neyns, B.; Hoeller, C.; Khushalani, N.I.; Miller, W.H., Jr.; Lao, C.D.; et al. Nivolumab versus chemotherapy in patients with advanced melanoma who progressed after anti-CTLA-4 treatment (CheckMate 037): A randomised, controlled, open-label, phase 3 trial. Lancet Oncol. 2015, 16, 375-384. [CrossRef]

98. Postow, M.A.; Chesney, J.; Pavlick, A.C.; Robert, C.; Grossmann, K.; McDermott, D.; Linette, G.P.; Meyer, N.; Giguere, J.K.; Agarwala, S.S.; et al. Nivolumab and ipilimumab versus ipilimumab in untreated melanoma. N. Engl. J. Med. 2015, 372, $2006-2017$. [CrossRef] [PubMed]

99. Gutzmer, R.; Stroyakovskiy, D.; Gogas, H.; Robert, C.; Lewis, K.; Protsenko, S.; Pereira, R.P.; Eigentler, T.; Rutkowski, P.; Demidov, L.; et al. Atezolizumab, vemurafenib, and cobimetinib as first-line treatment for unresectable advanced BRAFV600 mutationpositive melanoma (IMspire150): Primary analysis of the randomised, double-blind, placebo-controlled, phase 3 trial. Lancet 2020, 395, 1835-1844. [CrossRef]

100. Hatzivassiliou, G.; Song, K.; Yen, I.; Brandhuber, B.J.; Anderson, D.J.; Alvarado, R.; Ludlam, M.J.; Stokow, D.; Gloor, S.L.; Vigers, G.; et al. RAF inhibitors prime wild-type RAF to activate the MAPK pathway and enhance growth. Nature 2010, 464, 431-435. [CrossRef] [PubMed]

101. Menzer, C.; Menzies, A.M.; Carlino, M.S.; Reijers, I.; Groen, E.J.; Eigentler, T.; de Groot, J.W.B.; van der Veldt, A.A.M.; Johnson, D.B.; Meiss, F.; et al. Targeted Therapy in Advanced Melanoma with Rare BRAF Mutations. J. Clin. Oncol 2019, 37, $3142-3151$. [CrossRef] [PubMed]

102. Owsley, J.; Stein, M.K.; Porter, J.; In, G.K.; Salem, M.; O’Day, S.; Elliott, A.; Poorman, K.; Gibney, G.; VanderWalde, A. Prevalence of class I-III BRAF mutations among 114,662 cancer patients in a large genomic database. Exp. Biol Med. 2021, 246, 31-39. [CrossRef] [PubMed]

103. Planchard, D.; Besse, B.; Groen, H.J.M.; Souquet, P.J.; Quoix, E.; Baik, C.S.; Barlesi, F.; Kim, T.M.; Mazieres, J.; Novello, S.; et al. Dabrafenib plus trametinib in patients with previously treated BRAF(V600E)-mutant metastatic non-small cell lung cancer: An open-label, multicentre phase 2 trial. Lancet Oncol. 2017, 17, 984-993. [CrossRef] 
104. Planchard, D.; Kim, T.M.; Mazieres, J.; Quoix, E.; Riely, G.; Barlesi, F.; Souquet, P.J.; Smit, E.F.; Groen, H.J.; Kelly, R.J.; et al. Dabrafenib in patients with BRAF(V600E)-positive advanced non-small-cell lung cancer: A single-arm, multicentre, open-label, phase 2 trial. Lancet Oncol. 2016, 17, 642-650. [CrossRef]

105. Planchard, D.; Smit, E.F.; Groen, H.J.M.; Mazieres, J.; Besse, B.; Helland, Å.; Giannone, V.; D’ Amilio, A.M., Jr.; Zhang, P.; Mookerjee, B.; et al. Dabrafenib plus trametinib in patients with previously untreated BRAFV600E-mutant metastatic non-small-cell lung cancer: An open-label, phase 2 trial. Lancet Oncol. 2017, 18, 1307-1316. [CrossRef]

106. Facchinetti, F.; Lacroix, L.; Mezquita, L.; Scoazec, J.Y.; Loriot, Y.; Tselikas, L.; Gazzah, A.; Rouleau, E.; Adam, J.; Michiels, S.; et al. Molecular mechanisms of resistance to BRAF and MEK inhibitors in BRAFV600E non-small cell lung cancer. Eur. J. Cancer 2020, 132, 211-223. [CrossRef] [PubMed]

107. Yarchoan, M.; LiVolsi, V.A.; Brose, M.S. BRAF mutation and thyroid cancer recurrence. J. Clin. Oncol. 2015, 33, 7-8. [CrossRef]

108. Liu, R.; Bishop, J.; Zhu, G.; Zhang, T.; Ladenson, P.W.; Xin, M. Mortality Risk Stratification by Combining BRAF V600E and TERT Promoter Mutations in Papillary Thyroid Cancer: Genetic Duet of BRAF and TERT Promoter Mutations in Thyroid Cancer Mortality. JAMA Oncol. 2017, 3, 202-208. [CrossRef]

109. Brose, M.S.; Cabanillas, M.E.; Cohen, E.E.; Wirth, L.J.; Riehl, T.; Yue, H.; Sherman, S.I.; Sherman, E.J. Vemurafenib in patients with $\mathrm{BRAF}(\mathrm{V} 600 \mathrm{E})$-positive metastatic or unresectable papillary thyroid cancer refractory to radioactive iodine: A non-randomised, multicentre, open-label, phase 2 trial. Lancet Oncol. 2016, 17, 1272-1282. [CrossRef]

110. Falchook, G.S.; Millward, M.; Hong, D.; Naing, A.; Piha-Paul, S.; Waguespack, S.G.; Cabanillas, M.E.; Sherman, S.I.; Ma, B.; Curtis, M.; et al. BRAF inhibitor dabrafenib in patients with metastatic BRAF-mutant thyroid cancer. Thyroid 2015, 25, 71-77. [CrossRef]

111. Untch, B.R.; Olson, J.A., Jr. Anaplastic thyroid carcinoma, thyroid lymphoma, and metastasis to thyroid. Surg. Oncol. Clin. N. Am. 2006, 15, 661-679. [CrossRef] [PubMed]

112. Guerra, A.; Di Crescenzo, V.; Garzi, A.; Cinelli, M.; Carlomagno, C.; Tonacchera, M.; Zeppa, P.; Vitale, M. Genetic mutations in the treatment of anaplastic thyroid cancer: A systematic review. BMC Surg. 2013, 13 (Suppl. 2), S44. [CrossRef]

113. Subbiah, V.; Kreitman, R.J.; Wainberg, Z.A.; Cho, J.Y.; Schellens, J.H.M.; Soria, J.C.; Wen, P.Y.; Zielinski, C.; Cabanillas, M.E.; Urbanowitz, G.; et al. Dabrafenib and Trametinib Treatment in Patients with Locally Advanced or Metastatic BRAF V600-Mutant Anaplastic Thyroid Cancer. J. Clin. Oncol. 2018, 36, 7-13. [CrossRef] [PubMed]

114. Kopetz, S.; Grothey, A.; Yaeger, R.; van Cutsem, E.; Desai, J.; Yoshino, T.; Wasan, H.; Ciardiello, F.; Loupakis, F.; Hong, Y.S.; et al. Encorafenib, Binimetinib, and Cetuximab in BRAF V600E-Mutated Colorectal Cancer. N. Engl. J. Med. 2019, 381, 1632-1643. [CrossRef] [PubMed]

115. Tabernero, J.; Grothey, A.; van Cutsem, E.; Yaeger, R.; Wasan, H.; Yoshino, T.; Desai, J.; Ciardiello, F.; Loupakis, F.; Hong, Y.S.; et al. Encorafenib Plus Cetuximab as a New Standard of Care for Previously Treated BRAF V600E-Mutant Metastatic Colorectal Cancer: Updated Survival Results and Subgroup Analyses from the BEACON Study. J. Clin. Oncol. 2021, 39, 273-284. [CrossRef]

116. Cronin, K.A.; Harlan, L.C.; Dodd, K.W.; Abrams, J.S.; Ballard-Barbash, R. Population-based estimate of the prevalence of HER-2 positive breast cancer tumors for early stage patients in the US. Cancer Invest. 2010, 28, 963-968. [CrossRef]

117. Gordon, M.A.; Gundacker, H.M.; Benedetti, J.; Macdonald, J.S.; Baranda, J.C.; Levin, W.J.; Blanke, C.D.; Elatre, W.; Weng, P.; Zhou, J.Y.; et al. Assessment of HER2 gene amplification in adenocarcinomas of the stomach or gastroesophageal junction in the INT-0116/SWOG9008 clinical trial. Ann. Oncol. 2013, 24, 1754-1761. [CrossRef]

118. Slamon, D.J.; Clark, G.M.; Wong, S.G.; Levin, W.J.; Ullrich, A.; McGuire, W.L. Human breast cancer: Correlation of relapse and survival with amplification of the HER-2/neu oncogene. Science 1987, 235, 177-182. [CrossRef] [PubMed]

119. Lei, Y.Y.; Huang, J.Y.; Zhao, Q.R.; Jiang, N.; Xu, H.M.; Wang, Z.N.; Li, H.Q.; Zhang, S.B.; Sun, Z. The clinicopathological parameters and prognostic significance of HER2 expression in gastric cancer patients: A meta-analysis of literature. World J. Surg Oncol. 2017, 15, 68. [CrossRef] [PubMed]

120. Esteva, F.J.; Valero, V.; Booser, D.; Guerra, L.T.; Murray, J.L.; Pusztai, L.; Cristofanilli, M.; Arun, B.; Esmaeli, B.; Fritsche, H.A.; et al. Phase II study of weekly docetaxel and trastuzumab for patients with HER-2-overexpressing metastatic breast cancer. J. Clin. Oncol. 2002, 20, 1800-1808. [CrossRef]

121. Marty, M.; Cognetti, F.; Maraninchi, D.; Snyder, R.; Mauriac, L.; Tubiana-Hulin, M.; Chan, S.; Grimes, D.; Anton, A.; Lluch, A.; et al. Randomized phase II trial of the efficacy and safety of trastuzumab combined with docetaxel in patients with human epidermal growth factor receptor 2-positive metastatic breast cancer administered as first-line treatment: The M77001 study group. J. Clin. Oncol. 2005, 23, 4265-4274. [CrossRef]

122. Seidman, A.D.; Berry, D.; Cirrincione, C.; Harris, L.; Muss, H.; Marcom, P.K.; Gipson, G.; Burstein, H.; Lake, D.; Shapiro, C.; et al Randomized phase III trial of weekly compared with every-3-weeks paclitaxel for metastatic breast cancer, with trastuzumab for all HER-2 overexpressors and random assignment to trastuzumab or not in HER-2 nonoverexpressors: Final results of Cancer and Leukemia Group B protocol 9840. J. Clin. Oncol. 2008, 26, 1642-1649. [PubMed]

123. Tolaney, S.M.; Barry, W.T.; Dang, C.T.; Yardley, D.A.; Moy, B.; Marcom, P.K.; Albain, K.S.; Rugo, H.S.; Ellis, M.; Shapira, I.; et al. Adjuvant paclitaxel and trastuzumab for node-negative, HER2-positive breast cancer. N. Engl. J. Med. 2015, 372, 134-141. [CrossRef] [PubMed]

124. Slamon, D.; Eiermann, W.; Robert, N.; Pienkowski, T.; Martin, M.; Press, M.; Mackey, J.; Glaspy, J.; Chan, A.; Pawlicki, M.; et al. Adjuvant trastuzumab in HER2-positive breast cancer. N. Engl. J. Med. 2011, 365, 1273-1283. [CrossRef] [PubMed] 
125. Romond, E.H.; Perez, E.A.; Bryant, J.; Suman, V.J.; Geyer, C.E., Jr.; Davidson, N.E.; Tan-Chiu, E.; Martino, S.; Paik, S.; Kaufman, P.A.; et al. Trastuzumab plus adjuvant chemotherapy for operable HER2-positive breast cancer. N. Engl. J. Med. 2005, 353, 1673-1684. [CrossRef]

126. Geyer, C.E.; Forster, J.; Lindquist, D.; Chan, S.; Romieu, C.G.; Pienkowski, T.; Jagiello-Gruszfeld, A.; Crown, J.; Chan, A.; Kaufman, B.; et al. Lapatinib plus capecitabine for HER2-positive advanced breast cancer. N. Engl. J. Med. 2006, 355, 2733-2743. [CrossRef]

127. Blackwell, K.L.; Burstein, H.J.; Storniolo, A.M.; Rugo, H.; Sledge, G.; Koehler, M.; Ellis, C.; Casey, M.; Vukelja, S.; Bischoff, J.; et al. Randomized study of Lapatinib alone or in combination with trastuzumab in women with ErbB2-positive, trastuzumab-refractory metastatic breast cancer. J. Clin. Oncol. 2010, 28, 1124-1130. [CrossRef]

128. Baselga, J.; Cortés, J.; Kim, S.B.; Im, S.A.; Hegg, R.; Im, Y.H.; Roman, L.; Pedrini, J.L.; Pienkowski, T.; Knott, A.; et al. CLEOPATRA Study Group. Pertuzumab plus trastuzumab plus docetaxel for metastatic breast cancer. N. Engl. J. Med. 2012, 366, 109-119. [CrossRef] [PubMed]

129. Modi, S.; Saura, C.; Yamashita, T.; Park, Y.H.; Kim, S.B.; Tamura, K.; Andre, F.; Iwata, H.; Ito, Y.; Tsurutani, J.; et al. Trastuzumab Deruxtecan in Previously Treated HER2-Positive Breast Cancer. N. Engl. J. Med. 2020, 382, 610-621. [CrossRef] [PubMed]

130. Verma, S.; Miles, D.; Gianni, L.; Krop, I.E.; Welslau, M.; Baselga, J.; Pegram, M.; Oh, D.Y.; Dieras, V.; Guardino, E.; et al. Trastuzumab emtansine for HER2-positive advanced breast cancer. N. Engl. J. Med. 2012, 367, 1783-1791. [CrossRef]

131. Saura, C.; Oliveira, M.; Feng, Y.H.; Dai, M.S.; Chen, S.W.; Hurvitz, S.A.; Kim, S.B.; Moy, B.; Delaloge, S.; Gradishar, W.; et al. Neratinib Plus Capecitabine Versus Lapatinib Plus Capecitabine in HER2-Positive Metastatic Breast Cancer Previously Treated With $\geq 2$ HER2-Directed Regimens: Phase III NALA Trial. J. Clin. Oncol. 2020, 38, 3138-3149. [CrossRef] [PubMed]

132. Murthy, R.K.; Loi, S.; Okines, A.; Paplomata, E.; Hamilton, E.; Hurvitz, S.A.; Lin, N.U.; Borges, V.; Abramson, V.; Anders, C.; et al. Tucatinib, Trastuzumab, and Capecitabine for HER2-Positive Metastatic Breast Cancer. N. Engl. J. Med. 2020, 382, 597-609. [CrossRef]

133. Schneeweiss, A.; Chia, S.; Hickish, T.; Harvey, V.; Eniu, A.; Hegg, R.; Tausch, C.; Seo, J.H.; Tsai, Y.F.; Ratnayake, J.; et al. Pertuzumab plus trastuzumab in combination with standard neoadjuvant anthracycline-containing and anthracycline-free chemotherapy regimens in patients with HER2-positive early breast cancer. Ann. Oncol. 2013, 24, 2278-2284. [CrossRef]

134. Swain, S.M.; Ewer, M.S.; Viale, G.; Delaloge, S.; Ferrero, J.M.; Verrill, M.; Colomer, R.; Vieira, C.; Werner, T.L.; Douthwaite, H.; et al. Pertuzumab, trastuzumab, and standard anthracycline- and taxane-based chemotherapy for the neoadjuvant treatment of patients with HER2-positive localized breast cancer (BERENICE): A phase II, open-label, multicenter, multinational cardiac safety study. Ann. Oncol. 2018, 29, 646-653. [CrossRef]

135. Lin, N.U.; Borges, V.; Anders, C.; Murthy, R.K.; Paplomata, E.; Hamilton, E.; Hurvitz, S.; Loi, S.; Okines, A.; Abramson, V.; et al. Intracranial Efficacy and Survival with Tucatinib Plus Trastuzumab and Capecitabine for Previously Treated HER2-Positive Breast Cancer With Brain Metastases in the HER2CLIMB Trial. J. Clin. Oncol. 2020, 38, 2610-2619. [CrossRef]

136. Bang, Y.J.; van Cutsem, E.; Feyereislova, A.; Chung, H.C.; Shen, L.; Sawaki, A.; Lordick, F.; Ohtsu, A.; Omuro, Y.; Satoh, T.; et al. Trastuzumab in combination with chemotherapy versus chemotherapy alone for treatment of HER2-positive advanced gastric or gastro-oesophageal junction cancer (ToGA): A phase 3, open-label, randomised controlled trial. Lancet 2010, 376, 687-697. [CrossRef]

137. Shitara, K.; Bang, Y.J.; Iwasa, S.; Sugimoto, N.; Ryu, M.H.; Sakai, D.; Chung, H.C.; Kawakami, H.; Yabusaki, H.; Lee, J.; et al. Trastuzumab Deruxtecan in Previously Treated HER2-Positive Gastric Cancer. N. Engl. J. Med. 2020, 382, 2419-2430. [CrossRef]

138. Meric-Bernstam, F.; Hurwitz, H.; Raghav, K.P.S.; McWilliams, R.R.; Fakih, M.; VanderWalde, A.; Swanton, C.; Kurzrock, R.; Burris, H.; Sweeney, C.; et al. Pertuzumab plus trastuzumab for HER2-amplified metastatic colorectal cancer (MyPathway): An updated report from a multicentre, open-label, phase 2a, multiple basket study. Lancet Oncol. 2019, 20, 518-530. [CrossRef]

139. Nakamura, Y.; Okamoto, W.; Kato, T.; Hasegawa, H.; Kato, K.; Iwasa, S.; Esaki, T.; Komatsu, Y.; Masuishi, T.; Nishina, T.; et al TRIUMPH: Primary efficacy of a phase II trial of trastuzumab (T) and pertuzumab (P) in patients (pts) with metastatic colorectal cancer (mCRC) with HER2 (ERBB2) amplification (amp) in tumour tissue or circulating tumour DNA (ctDNA): A GOZILA sub-study. Ann. Oncol. 2019, 30 (Suppl. 5), v199-v200. [CrossRef]

140. Sartore-Bianchi, A.; Trusolino, L.; Martino, C.; Bencardino, K.; Lonardi, S.; Bergamo, F.; Zagonel, V.; Leone, F.; Depetris, I.; Martinelli, E.; et al. Dual-targeted therapy with trastuzumab and lapatinib in treatment-refractory, KRAS codon 12/13 wild-type, HER2-positive metastatic colorectal cancer (HERACLES): A proof-of-concept, multicentre, open-label, phase 2 trial. Lancet Oncol. 2016, 17, 738-746. [CrossRef]

141. Strickler, J.H.; Zemla, T.; Ou, F.-S.; Cercek, A.; Wu, C.; Sanchez, F.A.; Hubbard, J.; Jaszewski, B.; Bandel, L.; Schweitzer, B.; et al. Trastuzumab and tucatinib for the treatment of HER2 amplified metastatic colorectal cancer (mCRC): Initial results from the MOUNTAINEER trial. Ann. Oncol. 2019, 30 (Suppl. 5), v200. [CrossRef]

142. Sartore-Bianchi, A.; Lonardi, S.; Martino, C.; Fenocchio, E.; Tosi, F.; Ghezzi, S.; Leone, F.; Bergamo, F.; Zagonel, V.; Ciardiello, F.; et al. Pertuzumab and trastuzumab emtansine in patients with HER2-amplified metastatic colorectal cancer: The phase II HERACLES-B trial. ESMO Open 2020, 5, e000911. [CrossRef]

143. Siena, S.; Di Bartolomeo, M.; Raghav, K.; Masuishi, T.; Loupakis, F.; Kawakami, H.; Yamaguchi, K.; Nishina, T.; Faki, M.; Elez, E.; et al. Trastuzumab deruxtecan (DS-8201) in patients with HER2-expressing metastatic colorectal cancer (DESTINY-CRC01): A multicentre, open-label, phase 2 trial. Lancet Oncol. 2021, 4, S1470-S2045.

144. Hyman, D.M.; Piha-Paul, S.A.; Won, H.; Rodon, J.; Saura, C.; Shapiro, G.I.; Juric, D.; Quinn, D.I.; Moreno, V.; Doger, B.; et al. HER kinase inhibition in patients with HER2- and HER3-mutant cancers. Nature 2018, 554, 189-194. [CrossRef] [PubMed] 
145. Li, B.T.; Shen, R.; Buonocore, D.; Olah, Z.T.; Ni, A.; Ginsberg, M.S.; Ulaner, G.A.; Offin, M.; Feldman, D.; Hembrough, T.; et al. Ado-Trastuzumab Emtansine for Patients with HER2-Mutant Lung Cancers: Results From a Phase II Basket Trial. J. Clin. Oncol. 2018, 36, 2532-2537. [CrossRef] [PubMed]

146. Smit, E.F.; Nakagawa, K.; Nagasaka, M.; Felip, E.; Goto, Y.; Li, B.T.; Pacheco, J.M.; Murakami, H.; Barlesi, F.; Saltos, A.N.; et al. Trastuzumab deruxtecan (T-DXd; DS-8201) in patients with HER2-mutated metastatic non-small cell lung cancer (NSCLC): Interim results of DESTINY-Lung01. J. Clin. Oncol. 2020, 38 (Suppl.), 9504. [CrossRef]

147. Ciampi, R.; Romei, C.; Ramone, T.; Prete, A.; Tacito, A.; Cappagli, V.; Bottici, V.; Viola, D.; Torregrossa, L.; Ugolini, C.; et al. Genetic Landscape of Somatic Mutations in a Large Cohort of Sporadic Medullary Thyroid Carcinomas Studied by Next-Generation Targeted Sequencing. iScience 2019, 20, 324-336. [CrossRef] [PubMed]

148. Pozdeyev, N.; Gay, L.M.; Sokol, E.S.; Hartmaier, R.; Deaver, K.E.; Davis, S.; French, J.D.; Borre, P.V.; LaBarbera, D.V.; Tan, A.C.; et al. Genetic Analysis of 779 Advanced Differentiated and Anaplastic Thyroid Cancers. Clin. Cancer Res. 2018, 24, 3059-3068. [CrossRef] [PubMed]

149. Wang, R.; Hu, H.; Pan, Y.; Li, Y.; Ye, T.; Li, C.; Luo, X.; Wang, L.; Li, H.; Zhang, Y.; et al. RET fusions define a unique molecular and clinicopathologic subtype of non-small-cell lung cancer. J. Clin. Oncol. 2012, 30, 4352-4359. [CrossRef]

150. Drilon, A.; Oxnard, G.R.; Tan, D.S.W.; Loong, H.H.F.; Johnson, M.; Gainor, J.; McCoach, C.E.; Gautschi, O.; Besse, B.; Cho, B.C.; et al. Efficacy of Selpercatinib in RET Fusion-Positive Non-Small-Cell Lung Cancer. N. Engl. J. Med. 2020, 383, 813-824. [CrossRef]

151. Wirth, L.J.; Sherman, E.; Robinson, B.; Solomon, B.; Kang, H.; Lorch, J.; Worden, F.; Brose, M.; Patel, J.; Leboulleux, S.; et al. Efficacy of Selpercatinib in RET-Altered Thyroid Cancers. N. Engl. J. Med. 2020, 383, 825-835. [CrossRef]

152. Hu, M.; Subbiah, V.; Wirth, L.J.; Schuler, M.; Mansfield, A.S.; Brose, M.S.; Curigliano, G.; Leboulleux, S.; Zhu, V.W.; Keam, B.; et al. Results from the registrational phase I/II ARROW trial of pralsetinib (BLU-667) in patients (pts) with advanced RET mutation-positive medullary thyroid cancer (RET + MTC). Ann. Oncol. 2020, 31 (Suppl. 4), S1084. [CrossRef]

153. Gainor, J.F.; Curigliano, G.; Kim, D.-W.; Lee, D.H.; Besse, B.; Baik, C.S.; Doebele, R.C.; Cassier, P.A.; Lopez, G.; Tan, D.S.-W.; et al. Registrational dataset from the phase I/II ARROW trial of pralsetinib (BLU-667) in patients (pts) with advanced RET fusion+ non-small cell lung cancer (NSCLC). J. Clin. Oncol. 2020, 38, 9515. [CrossRef]

154. Zhu, V.W.; Madison, R.; Schrock, A.B.; Ou, S.I. Emergence of High Level of MET Amplification as Off-Target Resistance to Selpercatinib Treatment in KIF5B-RET NSCLC. J. Thorac. Oncol. 2020, 15, e124-e127. [CrossRef]

155. Lin, J.J.; Liu, S.V.; McCoach, C.E.; Zhu, V.W.; Tan, A.C.; Yoda, S.; Peterson, J.; Do, A.; Prutisto-Chang, K.; Dagogo-Jack, I.; et al. Mechanisms of resistance to selective RET tyrosine kinase inhibitors in RET fusion-positive non-small-cell lung cancer. Ann. Oncol. 2020, 31, 1725-1733. [CrossRef]

156. Moore, K.; Colombo, N.; Scambia, G.; Kim, B.G.; Oaknin, A.; Friedlander, M.; Lisyanskaya, A.; Floquet, A.; Leary, A.; Sonke, G.S.; et al. Maintenance Olaparib in Patients with Newly Diagnosed Advanced Ovarian Cancer. N. Engl. J. Med. 2018, 379, $2495-2505$. [CrossRef]

157. Ray-Coquard, I.; Pautier, P.; Pignata, S.; Pérol, D.; González-Martín, A.; Berger, R.; Fujiwara, K.; Vergote, I.; Colombo, N.; Mäenpää, J.; et al. Olaparib plus Bevacizumab as First-Line Maintenance in Ovarian Cancer. N. Engl. J. Med. 2019, 381, 2416-2428. [CrossRef] [PubMed]

158. Coleman, R.L.; Oza, A.M.; Lorusso, D.; Aghajanian, C.; Oaknin, A.; Dean, A.; Colombo, N.; Weberpals, J.I.; Clamp, A.; Scambia, G.; et al. Rucaparib maintenance treatment for recurrent ovarian carcinoma after response to platinum therapy (ARIEL3): A randomised, double-blind, placebo-controlled, phase 3 trial. Lancet 2017, 390, 1949-1961. [CrossRef]

159. González-Martín, A.; Pothuri, B.; Vergote, I.; DePont Christensen, R.; Graybill, W.; Mirza, M.R.; McCormick, C.; Lorusso, D.; Hoskins, P.; Freyer, G.; et al. Niraparib in Patients with Newly Diagnosed Advanced Ovarian Cancer. N. Engl. J. Med. 2019, 381, 2391-2402. [CrossRef]

160. de Bono, J.; Mateo, J.; Fizazi, K.; Saad, F.; Shore, N.; Sandhu, S.; Chi, K.N.; Sartor, O.; Agarwal, N.; Olmos, D.; et al. Olaparib for Metastatic Castration-Resistant Prostate Cancer. N. Engl. J. Med. 2020, 382, 2091-2102. [CrossRef]

161. Abida, W.; Patnaik, A.; Campbell, D.; Shapiro, J.; Bryce, A.H.; McDermott, R.; Sautois, B.; Vogelzang, N.J.; Bambury, R.M.; Voog, E.; et al. Rucaparib in Men with Metastatic Castration-Resistant Prostate Cancer Harboring a BRCA1 or BRCA2 Gene Alteration. J. Clin. Oncol. 2020, 38, 3763-3772. [CrossRef] [PubMed]

162. Robson, M.; Im, S.A.; Senkus, E.; Xu, B.; Domchek, S.M.; Masuda, N.; Delaloge, S.; Li, W.; Tung, N.; Armstrong, A.; et al. Olaparib for Metastatic Breast Cancer in Patients with a Germline BRCA Mutation. N. Engl. J. Med. 2017, 377, 523-533. [CrossRef] [PubMed]

163. Litton, J.K.; Rugo, H.S.; Ettl, J.; Hurvitz, S.A.; Gonçalves, A.; Lee, K.H.; Fehrenbacher, L.; Yerushalmi, R.; Mina, L.A.; Martin, M.; et al. Talazoparib in Patients with Advanced Breast Cancer and a Germline BRCA Mutation. N. Engl. J. Med. 2018, 379, 753-763. [CrossRef] [PubMed]

164. Holter, S.; Borgida, A.; Dodd, A.; Grant, R.; Semotiuk, K.; Hedley, D.; Dhani, N.; Narod, S.; Akbari, M.; Moore, M.; et al. Germline BRCA Mutations in a Large Clinic-Based Cohort of Patients with Pancreatic Adenocarcinoma. J. Clin. Oncol. 2015, 33, 3124-3129. [CrossRef] [PubMed]

165. Golan, T.; Hammel, P.; Reni, M.; van Cutsem, E.; Macarulla, T.; Hall, M.J.; Park, J.O.; Hochhauser, D.; Arnold, D.; Oh, D.Y.; et al. Maintenance Olaparib for Germline BRCA-Mutated Metastatic Pancreatic Cancer. N. Engl. J. Med. 2019, 381, 317-327. [CrossRef]

166. Keytruda Prescribing Information, Revised 02/2021. Available online: https://www.merck.com/product/usa/pi_circulars/k/ keytruda/keytruda_pi.pdf (accessed on 20 April 2021). 
167. Opdivo Prescribing Information. Revised 4/2021. Available online: https:/ / packageinserts.bms.com/pi/pi_opdivo.pdf (accessed on 20 April 2021).

168. Tecentriq Prescribing Information. Revised 04/21. Available online: https://www.gene.com/download/pdf/tecentriq prescribing.pdf (accessed on 20 April 2021).

169. Imfinzi Prescribing Information. Revised 02/21. Available online: https://den8dhaj6zs0e.cloudfront.net/50fd68b9-106b-4550-b5 d0-12b045f8b184/9496217c-08b3-432b-ab4f-538d795820bd/9496217c-08b3-432b-ab4f-538d795820bd_viewable_rendition_v. pdf (accessed on 20 April 2021).

170. Bavencio Prescribing Information. Revised 11/2020. Available online: https://www.emdserono.com/us-en/pi/bavencio-pi.pdf (accessed on 20 April 2021).

171. Libtayo Prescribing Information. Revised 02/2021. Available online: https://www.regeneron.com/downloads/libtayo_fpi.pdf (accessed on 20 April 2021).

172. Jemperli Prescribing Information. Revised 04/2021. Available online: https://www.accessdata.fda.gov/drugsatfda_docs/label/ 2021/761174s000lbl.pdf (accessed on 24 April 2021).

173. Patel, S.P.; Kurzrock, R. PD-L1 Expression as a Predictive Biomarker in Cancer Immunotherapy. Mol. Cancer Ther. 2015, 14, 847-856. [CrossRef]

174. Festino, L.; Botti, G.; Lorigan, P.; Masucci, G.V.; Hipp, J.D.; Horak, C.E.; Melero, I.; Ascierto, P.A. Cancer Treatment with Anti-PD-1/PD-L1 Agents: Is PD-L1 Expression a Biomarker for Patient Selection? Drugs 2016, 76, 925-945. [CrossRef] [PubMed]

175. Davis, A.A.; Patel, V.G. The role of PD-L1 expression as a predictive biomarker: An analysis of all US Food and Drug Administration (FDA) approvals of immune checkpoint inhibitors. J. Immunther. Cancer 2019, 7, 278. [CrossRef]

176. Update on Phase III DANUBE Trial for Imfinzi and Tremelimumab in Unresectable, Stage IV Bladder Cancer, Published 6 March 2020. Available online: https://www.astrazeneca.com/media-centre/press-releases/2020/update-on-phase-iii-danube-trialfor-imfinzi-and-tremelimumab-in-unresectable-stage-iv-bladder-cancer-06032020.html (accessed on 27 April 2021).

177. Powles, T.; van der Heijden, M.S.; Castellano, D.; Galsky, M.D.; Loriot, Y.; Petrylak, D.P.; Ogawa, O.; Park, S.H.; Lee, J.L.; De Giorgi, U.; et al. Durvalumab alone and durvalumab plus tremelimumab versus chemotherapy in previously untreated patients with unresectable, locally advanced or metastatic urothelial carcinoma (DANUBE): A randomised, open-label, multicentre, phase 3 trial. Lancet Oncol. 2020, 21, 1574-1588. [CrossRef]

178. FDA Expands Pembrolizumab Indication for First-Line Treatment of NSCLC (TPS $\geq 1 \%$ ). Content Current as of: $04 / 11 / 2019$. Available online: https:/ / www.fda.gov/drugs/fda-expands-pembrolizumab-indication-first-line-treatment-nsclc-tps-1 (accessed on 27 April 2021).

179. FDA Approves Nivolumab Plus Ipilimumab for First-Line mNSCLC (PD-L1 Tumor Expression $\geq 1 \%$ ). Content Current as of: 05/15/2020. Available online: https://www.fda.gov/drugs/drug-approvals-and-databases/fda-approves-nivolumab-plusipilimumab-first-line-mnsclc-pd-11-tumor-expression-1 (accessed on 27 April 2021).

180. FDA Approves Atezolizumab for First-Line Treatment of Metastatic NSCLC with High PD-L1 Expression. Content Current as of: 05/18/2020. Available online: https://www.fda.gov/drugs/resources-information-approved-drugs/fda-approvesatezolizumab-first-line-treatment-metastatic-nsclc-high-pd-11-expression (accessed on 27 April 2021).

181. FDA Approves Cemiplimab-Rwlc for Non-Small Cell Lung Cancer with High PD-L1 Expression. Content Current as of: 02/22/2021. Available online: https:/ / www.fda.gov/drugs/drug-approvals-and-databases/fda-approves-cemiplimab-rwlcnon-small-cell-lung-cancer-high-pd-11-expression (accessed on 27 April 2021).

182. FDA Approves Pembrolizumab for First-Line Treatment of Head and Neck Squamous Cell Carcinoma. Content Current as of: 06/11/2019. Available online: https://www.fda.gov/drugs/resources-information-approved-drugs/fda-approvespembrolizumab-first-line-treatment-head-and-neck-squamous-cell-carcinoma (accessed on 27 April 2021).

183. FDA Limits the Use of Tecentriq and Keytruda for Some Urothelial Cancer Patients. Content Current as of: 07/05/2018. Available online: https: / / www.fda.gov / drugs/resources-information-approved-drugs/fda-limits-use-tecentriq-and-keytruda-someurothelial-cancer-patients (accessed on 27 April 2021).

184. Fashoyin-Aje, L.; Donoghue, M.; Chen, H.; He, K.; Veeraraghavan, J.; Goldberg, K.B.; Keegan, P.; McKee, A.E.; Pazdur, R. FDA Approval Summary: Pembrolizumab for Recurrent Locally Advanced or Metastatic Gastric or Gastroesophageal Junction Adenocarcinoma Expressing PD-L1. Oncologist 2019, 24, 103-109. [CrossRef]

185. FDA Approves Pembrolizumab for Advanced Esophageal Squamous Cell Cancer. Content Current as of: 07/31/2019. Available online: https:/ / www.fda.gov/drugs/resources-information-approved-drugs/fda-approves-pembrolizumab-advancedesophageal-squamous-cell-cancer (accessed on 27 April 2021).

186. FDA Approves Pembrolizumab for Advanced Cervical Cancer with Disease Progression during or after Chemotherapy. Content Current as of: 06/13/2018. Available online: https://www.fda.gov/drugs/resources-information-approved-drugs/ fda-approves-pembrolizumab-advanced-cervical-cancer-disease-progression-during-or-after-chemotherapy (accessed on 27 April 2021).

187. Narayan, P.; Wahby, S.; Gao, J.J.; Amiri-Kordestani, L.; Ibrahim, A.; Bloomquist, E.; Tang, S.; Xu, Y.; Liu, J.; Fu, W.; et al. FDA Approval Summary: Atezolizumab Plus Paclitaxel Protein-bound for the Treatment of Patients with Advanced or Metastatic TNBC Whose Tumors Express PD-L1. Clin. Cancer Res. 2020, 26, 2284-2289. [CrossRef] 
188. FDA Grants Accelerated Approval to Pembrolizumab for Locally Recurrent Unresectable or Metastatic Triple Negative Breast Cancer. Content Current as of: 11/13/2020. Available online: https://www.fda.gov/drugs/drug-approvals-and-databases/fdagrants-accelerated-approval-pembrolizumab-locally-recurrent-unresectable-or-metastatic-triple (accessed on 27 April 2021 ).

189. Bar-Sagi, D.; Knelson, E.H.; Sequist, L.V. A bright future for KRAS inhibitors. Nat. Cancer 2020, 1, 25-27. [CrossRef]

190. Nadal, E.; Chen, G.; Prensner, J.R.; Shiratsuchi, H.; Sam, C.; Zhao, L.; Kalemkerian, G.P.; Brenner, D.; Lin, J.; Reddy, R.M.; et al. KRAS-G12C mutation is associated with poor outcome in surgically resected lung adenocarcinoma. J. Thorac. Oncol. 2014, 9, 1513-1522. [CrossRef] [PubMed]

191. Jones, R.P.; Sutton, P.A.; Evans, J.P.; Clifford, R.; McAvoy, A.; Lewis, J.; Rousseau, A.; Mountford, R.; McWhirter, D.; Malik, H.Z. Specific mutations in KRAS codon 12 are associated with worse overall survival in patients with advanced and recurrent colorectal cancer. Br. J. Cancer 2017, 116, 923-929. [CrossRef]

192. Del Re, M.; Rofi, E.; Restante, G.; Crucitta, S.; Arrigoni, E.; Fogli, S.; DiMaio, M.; Petrini, I.; Danesi, R. Implications of KRAS mutations in acquired resistance to treatment in NSCLC. Oncotarget 2017, 9, 6630-6643. [CrossRef]

193. Ostrem, J.M.; Peters, U.; Sos, M.L.; Wells, J.A.; Shokat, K.M. K-Ras(G12C) inhibitors allosterically control GTP affinity and effector interactions. Nature 2013, 503, 548-551. [CrossRef] [PubMed]

194. Canon, J.; Rex, K.; Saiki, A.Y.; Mohr, C.; Cooke, K.; Bagal, D.; Gaida, K.; Holt, T.; Knutson, C.G.; Koppada, N.; et al. The clinical KRAS(G12C) inhibitor AMG 510 drives anti-tumour immunity. Nature 2019, 575, 217-223. [CrossRef]

195. Hallin, J.; Engstrom, L.D.; Hargis, L.; Calinisan, A.; Aranda, R.; Briere, D.M.; Sudhakar, N.; Bowcut, V.; Baer, B.R.; Ballard, J.A.; et al. The KRASG12C Inhibitor MRTX849 Provides Insight toward Therapeutic Susceptibility of KRAS-Mutant Cancers in Mouse Models and Patients. Cancer Discov. 2020, 10, 54-71. [CrossRef] [PubMed]

196. Hong, D.S.; Fakih, M.G.; Strickler, J.H.; Desai, J.; Durm, G.A.; Shapiro, G.I.; Falchook, G.S.; Price, T.J.; Sacher, A.; Denlinger, C.S.; et al. KRASG12C Inhibition with Sotorasib in Advanced Solid Tumors. N. Engl. J. Med. 2020, 383, 1207-1217. [CrossRef] [PubMed]

197. LoRusso, P.M.; Sebolt-Leopold, J.S. One Step at a Time-Clinical Evidence That KRAS Is Indeed Druggable. N. Engl. J. Med. 2020, 383, 1277-1278. [CrossRef]

198. Amodio, V.; Yaeger, R.; Arcella, P.; Cancelliere, C.; Lamba, S.; Lorenzato, A.; Arena, S.; Montone, M.; Mussolin, B.; Bian, Y.; et al EGFR Blockade Reverts Resistance to KRASG12C Inhibition in Colorectal Cancer. Cancer Discov. 2020, 10, 1129-1139. [CrossRef]

199. Misale, S.; Fatherree, J.P.; Cortez, E.; Li, C.; Bilton, S.; Timonina, D.; Myers, D.T.; Lee, D.; Gomez-Caraballo, M.; Greenberg, M.; et al. KRAS G12C NSCLC Models Are Sensitive to Direct Targeting of KRAS in Combination with PI3K Inhibition. Clin. Cancer Res. 2019, 25, 796-807. [CrossRef]

200. Riely, G.J.; Ou, S.-H.I.; Rybkin, I.; Spira, A.; Papadopoulos, K.; Sabari, J.K.; Johnson, M.; Heist, R.S.; Bazhenova, L.; Barve, M.; et al. KRYSTAL-1: Activity and preliminary pharmacodynamic (PD) analysis of adagrasib (MRTX849) in patients (Pts) with advanced non-small cell lung cancer (NSCLC) harboring KRASG12C mutation. J. Thorac. Oncol. 2021, 16, S751-S752. [CrossRef]

201. Siegel, R.L.; Miller, K.D.; Jemal, A. Cancer Statistics, 2020. CA Cancer J. Clin. 2020, 70, 7-30. [CrossRef]

202. Noone, A.M.; Cronin, K.A.; Altekruse, S.F.; Howlader, N.; Lewis, D.R.; Petkov, V.I.; Penberthy, L. Cancer Incidence and Survival Trends by Subtype Using Data from the Surveillance Epidemiology and End Results Program, 1992-2013. Cancer Epidemiol. Biomark. Prev. 2017, 26, 632-641. [CrossRef] [PubMed]

203. Howlader, N.; Forjaz, G.; Mooradian, M.J.; Meza, R.; Kong, C.Y.; Cronin, K.A.; Mariotto, A.B.; Lowy, D.R.; Feuer, E.J. The Effect of Advances in Lung-Cancer Treatment on Population Mortality. N. Engl. J. Med. 2020, 383, 640-649. [CrossRef]

204. National Comprehensive Cancer Network. Non-Small Cell Lung Cancer (Version 4.2021-March 3, 2021). Available online: https:/ / www.nccn.org/professionals/physician_gls/pdf/nscl.pdf (accessed on 22 April 2021).

205. Lindeman, N.I.; Cagle, P.T.; Aisner, D.L.; Arcila, M.E.; Beasley, M.B.; Bernicker, E.H.; Colasacco, C.; Dacic, S.; Hirsch, F.R.; Kerr, K.; et al. Updated Molecular Testing Guideline for the Selection of Lung Cancer Patients for Treatment with Targeted Tyrosine Kinase Inhibitors: Guideline From the College of American Pathologists, the International Association for the Study of Lung Cancer, and the Association for Molecular Pathology. J. Mol. Diagn. 2018, 20, 129-159.

206. Rolfo, C.; Mack, P.C.; Scagliotti, G.V.; Baas, P.; Barlesi, F.; Bivona, T.G.; Herbst, R.S.; Mok, T.S.; Peled, N.; Pirker, R.; et al. Liquid Biopsy for Advanced Non-Small Cell Lung Cancer (NSCLC): A Statement Paper from the IASLC. J. Thorac. Oncol. 2018, 13, 1248-1268. [CrossRef]

207. Leighl, N.B.; Page, R.D.; Raymond, V.M.; Daniel, D.B.; Divers, S.G.; Reckamp, K.L.; Villalona-Calero, M.S.; Dix, D.; Odegaard, J.I.; Lanman, R.B.; et al. Clinical Utility of Comprehensive Cell-free DNA Analysis to Identify Genomic Biomarkers in Patients with Newly Diagnosed Metastatic Non-Small Cell Lung Cancer. Clin. Cancer Res. 2019, 25, 4691-4700. [CrossRef]

208. Schoenfeld, A.J.; Arbour, K.C.; Rizvi, H.; Iqbal, A.N.; Gadgeel, S.M.; Girshman, J.; Kris, M.G.; Riely, G.J.; Yu, H.A.; Hellmann, M.D. Severe immune-related adverse events are common with sequential PD-(L)1 blockade and osimertinib. Ann. Oncol. 2019, 30, 839-844. [CrossRef]

209. Spigel, D.R.; Reynolds, C.; Waterhouse, D.; Garon, E.B.; Chandler, J.; Babu, S.; Thurmes, P.; Spira, A.; Jotte, R.; Zhu, J.; et al. Phase $1 / 2$ Study of the Safety and Tolerability of Nivolumab Plus Crizotinib for the First-Line Treatment of Anaplastic Lymphoma Kinase Translocation-Positive Advanced Non-Small Cell Lung Cancer (CheckMate 370). J. Thorac. Oncol. 2018, 13, 682-688. [CrossRef]

210. Khozin, S.; Blumenthal, G.M.; Jiang, X.; He, K.; Boyd, K.; Murgo, A.; Justice, R.; Keegan, P.; Pazdur, R.U.S. Food and Drug Administration Approval Summary: Erlotinib for the First-Line Treatment of Metastatic Non-Small Cell Lung Cancer with 
Epidermal Growth Factor Receptor Exon 19 Deletions or Exon 21 (L858R) Substitution Mutations. Oncologist 2014, 19, 774-779. [CrossRef]

211. Kazandjian, D.; Blumenthal, G.M.; Yuan, W.; He, K.; Keegan, P.; Pazdur, R. FDA Approval of Gefitinib for the Treatment of Patients with Metastatic EGFR Mutation-Positive Non-Small Cell Lung Cancer. Clin. Cancer Res. 2016, 22, 1307-1312. [CrossRef]

212. FDA Broadens Afatinib Indication to Previously Untreated, Metastatic NSCLC with Other Non-Resistant EGFR Mutations Content Current as of: 01/16/2018. Available online: https:/ / www.fda.gov/drugs/resources-information-approved-drugs/fdabroadens-afatinib-indication-previously-untreated-metastatic-nsclc-other-non-resistant-egfr (accessed on 26 April 2021).

213. FDA Approves Dacomitinib for Metastatic Non-Small Cell Lung Cancer. Content Current as of: 11/26/2018. Available online: https: / / www.fda.gov/drugs/drug-approvals-and-databases/fda-approves-dacomitinib-metastatic-non-small-celllung-cancer (accessed on 26 April 2021).

214. FDA Approves Osimertinib for First-Line Treatment of Metastatic NSCLC with Most Common EGFR Mutations. Content current as of: 04/19/2018. Available online: https://www.fda.gov/drugs/resources-information-approved-drugs/fda-approvesosimertinib-first-line-treatment-metastatic-nsclc-most-common-egfr-mutations (accessed on 26 April 2021).

215. FDA Approves Osimertinib as Adjuvant Therapy for Non-Small Cell Lung Cancer with EGFR Mutations. Available online: https: / www.fda.gov/drugs/drug-approvals-and-databases/fda-approves-osimertinib-adjuvant-therapy-non-smallcell-lung-cancer-egfr-mutations (accessed on 23 April 2021).

216. FDA Grants Accelerated Approval to Amivantamab-Vmjw for Metastatic Non-Small Cell Lung Cancer. Content Current as of 05/21/2021. Available online: https:/ / www.fda.gov/drugs/drug-approvals-and-databases/fda-grants-accelerated-approvalamivantamab-vmjw-metastatic-non-small-cell-lung-cancer (accessed on 26 May 2021).

217. Kazandjian, D.; Blumenthal, G.M.; Chen, H.Y.; He, K.; Patel, M.; Justice, R.; Keegan, P.; Pazdur, R. FDA approval summary: Crizotinib for the treatment of metastatic non-small cell lung cancer with anaplastic lymphoma kinase rearrangements. Oncologist 2014, 19, e5-e11. [CrossRef] [PubMed]

218. Khozin, S.; Blumenthal, G.M.; Zhang, L.; Tang, S.; Brower, M.; Fox, E.; Helms, W.; Leong, R.; Song, P.; Pan, Y.; et al. FDA approval: Ceritinib for the treatment of metastatic anaplastic lymphoma kinase-positive non-small cell lung cancer. Clin. Cancer Res. 2015, 21, 2436-2439. [CrossRef] [PubMed]

219. FDA Approves Lorlatinib for Second- or Third-Line Treatment of ALK-Positive Metastatic NSCLC. Content Current as of: 12/14/2018. Available online: https:/ / www.fda.gov/drugs/fda-approves-lorlatinib-second-or-third-line-treatment-alkpositive-metastatic-nsclc (accessed on 26 April 2021).

220. Larkins, E.; Blumenthal, G.M.; Chen, H.; He, K.; Agarwal, R.; Gieser, G.; Stephens, O.; Zahalka, E.; Ringgold, K.; Helms, W.; et al. FDA Approval: Alectinib for the Treatment of Metastatic, ALK-Positive Non-Small Cell Lung Cancer Following Crizotinib. Clin. Cancer Res. 2016, 22, 5171-5176. [CrossRef] [PubMed]

221. Brigatinib. Content Current as of: 07/25/2017. Available online: https:/ /www.fda.gov/drugs/resources-information-approveddrugs/brigatinib (accessed on 26 April 2021).

222. Kazandjian, D.; Blumenthal, G.M.; Luo, L.; He, K.; Fran, I.; Lemery, S.; Pazdur, R. Benefit-Risk Summary of Crizotinib for the Treatment of Patients with ROS1 Alteration-Positive, Metastatic Non-Small Cell Lung Cancer. Oncologist 2016, 21, 974-980. [CrossRef] [PubMed]

223. FDA Grants Accelerated Approval to Capmatinib for Metastatic Non-Small Cell Lung Cancer. Content Current as of: 05/06/2020. Available online: https://www.fda.gov/drugs/drug-approvals-and-databases/fda-grants-accelerated-approval-capmatinibmetastatic-non-small-cell-lung-cancer (accessed on 26 April 2021).

224. FDA Grants Accelerated Approval to Tepotinib for Metastatic Non-Small Cell Lung Cancer. Content Current as of: 02/03/2021. Available online: https://www.fda.gov/drugs/drug-approvals-and-databases/fda-grants-accelerated-approval-tepotinibmetastatic-non-small-cell-lung-cancer (accessed on 26 April 2021).

225. Hirsch, F.R.; Bunn, P.A., Jr. EGFR testing in lung cancer is ready for prime time. Lancet Oncol. 2009, 10, 432-433. [CrossRef]

226. O'Kane, G.M.; Bradbury, P.A.; Feld, R.; Leighl, N.B.; Liu, G.; Pisters, K.M.; Kamel-Reid, S.; Tsao, M.S.; Shepherd, F.A. Uncommon EGFR mutations in advanced non-small cell lung cancer. Lung Cancer 2017, 109, 137-144. [CrossRef]

227. Mok, T.S.; Wu, Y.L.; Thongprasert, S.; Yang, C.H.; Chu, D.T.; Saijo, N.; Sunpaweravong, P.; Han, B.; Margono, B.; Ichinose, Y.; et al. Gefitinib or carboplatin-paclitaxel in pulmonary adenocarcinoma. N. Engl. J. Med. 2009, 361, 947-957. [CrossRef]

228. Fukuoka, M.; Wu, Y.L.; Thongprasert, S.; Sunpaweravong, P.; Leong, S.S.; Sriuranpong, V.; Chao, T.Y.; Nakagawa, K.; Chu, D.T.; Saijo, N.; et al. Biomarker analyses and final overall survival results from a phase III, randomized, open-label, first-line study of gefitinib versus carboplatin/paclitaxel in clinically selected patients with advanced non-small-cell lung cancer in Asia (IPASS). J. Clin. Oncol. 2011, 29, 2866-2874. [CrossRef]

229. Douillard, J.Y.; Ostoros, G.; Cobo, M.; Ciuleanu, T.; McCormack, R.; Webster, A.; Milenkova, T. First-line gefitinib in Caucasian EGFR mutation-positive NSCLC patients: A phase-IV, open-label, single-arm study. Br. J. Cancer 2014, 110, 55-62. [CrossRef]

230. Rosell, R.; Carcereny, E.; Gervais, R.; Vergnenegre, A.; Massuti, B.; Felip, E.; Palmero, R.; Garcia-Gomez, R.; Pallares, C.; Sanchez, J.M.; et al. Erlotinib versus standard chemotherapy as first-line treatment for European patients with advanced EGFR mutationpositive non-small-cell lung cancer (EURTAC): A multicentre, open-label, randomised phase 3 trial. Lancet Oncol. 2012, 13, 239-246. [CrossRef] 
231. Nakagawa, K.; Garon, E.B.; Seto, T.; Nishio, M.; Ponce Aix, S.; Paz-Ares, L.; Chiu, C.H.; Park, K.; Novello, S.; Nadal, E.; et al. Ramucirumab plus erlotinib in patients with untreated, EGFR-mutated, advanced non-small-cell lung cancer (RELAY): A randomised, double-blind, placebo-controlled, phase 3 trial. Lancet Oncol. 2019, 20, 1655-1669. [CrossRef]

232. Saito, H.; Fukuhara, T.; Furuya, N.; Watanabe, K.; Sugawara, S.; Iwasawa, S.; Tsunezuka, Y.; Yamaguchi, O.; Okada, M.; Yoshimori, K.; et al. Erlotinib plus bevacizumab versus erlotinib alone in patients with EGFR-positive advanced non-squamous non-small-cell lung cancer (NEJ026): Interim analysis of an open-label, randomised, multicentre, phase 3 trial. Lancet Oncol. 2019, 20, 625-635. [CrossRef]

233. Yang, J.C.; Sequist, L.V.; Geater, S.L.; Tsai, C.M.; Mok, T.S.; Schuler, M.; Yamamoto, N.; Yu, C.J.; Ou, S.H.; Zhou, C.; et al. Clinical activity of afatinib in patients with advanced non-small-cell lung cancer harbouring uncommon EGFR mutations: A combined post-hoc analysis of LUX-Lung 2, LUX-Lung 3, and LUX-Lung 6. Lancet Oncol. 2015, 16, 830-838. [CrossRef]

234. Yang, J.C.; Schuler, M.; Popat, S.; Miura, S.; Heeke, S.; Park, K.; Märten, A.; Kim, E.S. Afatinib for the Treatment of NSCLC Harboring Uncommon EGFR Mutations: A Database of 693 Cases. J. Thorac. Oncol. 2020, 15, 803-815. [CrossRef]

235. Wu, Y.L.; Cheng, Y.; Zhou, X.; Lee, K.H.; Nakagawa, K.; Niho, S.; Tsuji, F.; Linke, R.; Rosell, R.; Corral, J.; et al. Dacomitinib versus gefitinib as first-line treatment for patients with EGFR-mutation-positive non-small-cell lung cancer (ARCHER 1050): A randomised, open-label, phase 3 trial. Lancet Oncol. 2017, 18, 1454-1466. [CrossRef]

236. Mok, T.S.; Cheng, Y.; Zhou, X.; Lee, K.H.; Nakagawa, K.; Niho, S.; Lee, M.; Linke, R.; Rosell, R.; Corral, J.; et al. Improvement in Overall Survival in a Randomized Study That Compared Dacomitinib With Gefitinib in Patients with Advanced Non-Small-Cell Lung Cancer and EGFR-Activating Mutations. J. Clin. Oncol. 2018, 36, 2244-2250. [CrossRef] [PubMed]

237. Mok, T.S.; Wu, Y.-L.; Ahn, M.-J.; Garassino, M.C.; Kim, H.R.; Ramalingam, S.S.; Shepherd, F.A.; He, Y.; Akamatsu, H.; Theelen, W.S.; et al. Osimertinib or Platinum-Pemetrexed in EGFR T790M-Positive Lung Cancer. N. Engl. J. Med. 2017, 376, 629-640. [CrossRef]

238. Soria, J.C.; Ohe, Y.; Vansteenkiste, J.; Reungwetwattana, T.; Chewaskulyong, B.; Lee, K.H.; Dechaphunkul, A.; Imamura, F.; Nogami, N.; Kurata, T.; et al. Osimertinib in Untreated EGFR-Mutated Advanced Non-Small-Cell Lung Cancer. N. Engl. J. Med. 2018, 378, 113-125. [CrossRef] [PubMed]

239. Ramalingam, S.S.; Vansteenkiste, J.; Planchard, D.; Cho, B.C.; Gray, J.E.; Ohe, Y.; Zhou, C.; Reungwetwattana, T.; Cheng, Y.; Chewaskulyong, B.; et al. Overall Survival with Osimertinib in Untreated, EGFR-Mutated Advanced NSCLC. N. Engl. J. Med. 2020, 382, 41-50. [CrossRef] [PubMed]

240. Reungwetwattana, T.; Nakagawa, K.; Cho, B.C.; Cobo, M.; Cho, E.K.; Bertolini, A.; Bohnet, S.; Zhou, C.; Lee, K.H.; Nogami, N.; et al. CNS Response to Osimertinib Versus Standard Epidermal Growth Factor Receptor Tyrosine Kinase Inhibitors in Patients with Untreated EGFR-Mutated Advanced Non-Small-Cell Lung Cancer. J. Clin. Oncol. 2018, 36, 3290-3297. [CrossRef]

241. Yang, J.C.H.; Kim, S.W.; Kim, D.W.; Lee, J.S.; Cho, B.C.; Ahn, J.S.; Lee, D.H.; Kim, T.M.; Goldman, J.W.; Natale, R.B.; et al. Osimertinib in Patients with Epidermal Growth Factor Receptor Mutation-Positive Non-Small-Cell Lung Cancer and Leptomeningeal Metastases: The BLOOM Study. J. Clin. Oncol. 2020, 38, 538-547. [CrossRef]

242. Coleman, N.; Woolf, D.; Welsh, L.; McDonald, F.; MacMahon, S.; Yousaf, N.; Popat, S. EGFR Exon 20 Insertion (A763_Y764insFQEA) Mutant NSCLC Is Not Identified by Roche Cobas Version 2 Tissue Testing but Has Durable Intracranial and Extracranial Response to Osimertinib. J. Thorac. Oncol. 2020, 15, e162-e165. [CrossRef] [PubMed]

243. Sehgal, K.; Rangachari, D.; VanderLaan, P.A.; Kobayashi, S.S.; Costa, D.B. Clinical Benefit of Tyrosine Kinase Inhibitors in Advanced Lung Cancer with EGFR-G719A and Other Uncommon EGFR Mutations. Oncologist 2021, 26, 281-287. [CrossRef]

244. Wu, Y.L.; Tsuboi, M.; He, J.; John, T.; Grohe, C.; Majem, M.; Goldman, J.W.; Laktionov, K.; Kim, S.W.; Kato, T.; et al. Osimertinib in Resected EGFR-Mutated Non-Small-Cell Lung Cancer. N. Engl. J. Med. 2020, 383, 1711-1723. [CrossRef]

245. Piper-Vallillo, A.J.; Sequist, L.V.; Piotrowska, Z. Emerging Treatment Paradigms for EGFR-Mutant Lung Cancers Progressing on Osimertinib: A Review. J. Clin. Oncol. 2020, 38, 2926-2936. [CrossRef]

246. Sabari, J.K.; Shu, C.A.; Park, K.; Leighl, N.; Mitchell, P.; Kim, S.; Lee, J.; Kim, D.; Viteri, S.; Spira, A.; et al. Amivantamab in Post-platinum EGFR Exon 20 Insertion Mutant Non-Small Cell Lung Cancer. J. Thorac. Oncol. 2021, 16, S108-S109. [CrossRef]

247. Zhou, C.; Ramalingam, S.; Li, B.; Fang, J.; Kim, T.M.; Kim, S.; Yang, J.C.; Riely, J.; Mekhail, T.; Nguyen, D.; et al. Mobocertinib in NSCLC With EGFR Exon 20 Insertions: Results from EXCLAIM and Pooled Platinum-Pretreated Patient Populations. J. Thorac. Oncol. 2021, 16, S108. [CrossRef]

248. Kwak, E.L.; Bang, Y.J.; Camidge, D.R.; Shaw, A.T.; Solomon, B.; Maki, R.G.; Ou, S.H.; Dezube, B.J.; Jänne, P.A.; Costa, D.B.; et al. Anaplastic lymphoma kinase inhibition in non-small-cell lung cancer. N. Engl. J. Med. 2010, 363, 1693-1703. [CrossRef] [PubMed]

249. Camidge, D.R.; Bang, Y.J.; Kwak, E.L.; Iafrate, A.J.; Varella-Garcia, M.; Fox, S.B.; Riely, G.J.; Solomon, B.; Ou, S.H.; Kim, D.W.; et al. Activity and safety of crizotinib in patients with ALK-positive non-small-cell lung cancer: Updated results from a phase 1 study. Lancet Oncol. 2012, 13, 1011-1019. [CrossRef]

250. Kim, D.-W.; Ahn, M.-J.; Shi, Y.; de Pas, T.M.; Yang, P.-C.; Riely, G.J.; Crino, L.; Evans, T.L.; Liu, X.; Han, J.-Y.; et al. Results of a global phase II study with crizotinib in advanced ALK-positive non-small cell lung cancer (NSCLC). J. Clin. Oncol. 2012, 30 (Suppl.), 7533. [CrossRef]

251. Shaw, A.T.; Ou, S.H.; Bang, Y.J.; Camidge, D.R.; Solomon, B.J.; Salgia, R.; Riely, G.J.; Varella-Garcia, M.; Shapiro, G.I.; Costa, D.B.; et al. Crizotinib in ROS1-rearranged non-small-cell lung cancer. N. Engl. J. Med. 2014, 371, 1963-1971. [CrossRef] [PubMed] 
252. Shaw, A.T.; Riely, G.J.; Bang, Y.J.; Kim, D.W.; Camidge, D.R.; Solomon, B.J.; Varella-Garcia, M.; Iafrate, A.J.; Shapiro, G.I.; Usari, T.; et al. Crizotinib in ROS1-rearranged advanced non-small-cell lung cancer (NSCLC): Updated results, including overall survival, from PROFILE 1001. Ann. Oncol. 2019, 30, 1121-1126. [CrossRef] [PubMed]

253. Soria, J.C.; Tan, D.S.W.; Chiari, R.; Wu, Y.L.; Paz-Ares, L.; Wolf, J.; Geater, S.L.; Orlov, S.; Cortinovis, D.; Yu, C.J.; et al. Firstline ceritinib versus platinum-based chemotherapy in advanced ALK-rearranged non-small-cell lung cancer (ASCEND-4): A randomised, open-label, phase 3 study. Lancet 2017, 389, 917-929. [CrossRef]

254. Shaw, A.T.; Felip, E.; Bauer, T.M.; Besse, B.; Navarro, A.; Postel-Vinay, S.; Gainor, J.F.; Johnson, M.; Dietrich, J.; James, L.P.; et al. Lorlatinib in non-small-cell lung cancer with ALK or ROS1 rearrangement: An international, multicentre, open-label, single-arm first-in-man phase 1 trial. Lancet Oncol. 2017, 18, 1590-1599. [CrossRef]

255. Shaw, A.T.; Bauer, T.M.; de Marinis, F.; Felip, E.; Goto, Y.; Liu, G.; Mazieres, J.; Kim, D.W.; Mok, T.; Polli, A.; et al. First-Line Lorlatinib or Crizotinib in Advanced ALK-Positive Lung Cancer. N. Engl. J. Med. 2020, 383, 2018-2029. [CrossRef] [PubMed]

256. Camidge, D.R.; Dziadziuszko, R.; Peters, S.; Mok, T.; Noe, J.; Nowicka, M.; Gadgeel, S.M.; Cheema, P.; Pavlakis, N.; de Marinis, F.; et al. Updated Efficacy and Safety Data and Impact of the EML4-ALK Fusion Variant on the Efficacy of Alectinib in Untreated ALK-Positive Advanced Non-Small Cell Lung Cancer in the Global Phase III ALEX Study. J. Thorac. Oncol. 2019, 14, 1233-1243. [CrossRef] [PubMed]

257. Mok, T.; Camidge, D.R.; Gadgeel, S.M.; Rosell, R.; Dziadziuszko, R.; Kim, D.W.; Perol, M.; Ou, S.I.; Ahn, J.S.; Shaw, A.T.; et al. Updated overall survival and final progression-free survival data for patients with treatment-naive advanced ALK-positive non-small-cell lung cancer in the ALEX study. Ann. Oncol. 2020, 31, 1056-1064. [CrossRef]

258. Kim, D.W.; Tiseo, M.; Ahn, M.J.; Reckamp, K.L.; Hansen, K.H.; Kim, S.W.; Huber, R.M.; West, H.L.; Groen, H.J.M.; Hochmair, M.J.; et al. Brigatinib in Patients with Crizotinib-Refractory Anaplastic Lymphoma Kinase-Positive Non-Small-Cell Lung Cancer: A Randomized, Multicenter Phase II Trial. J. Clin. Oncol. 2017, 35, 2490-2498. [CrossRef] [PubMed]

259. Huber, R.M.; Hansen, K.H.; Paz-Ares Rodríguez, L.; West, H.L.; Reckamp, K.L.; Leighl, N.B.; Tiseo, M.; Smit, E.F.; Kim, D.W.; Gettinger, S.N.; et al. Brigatinib in Crizotinib-Refractory ALK+ NSCLC: 2-Year Follow-up on Systemic and Intracranial Outcomes in the Phase 2 ALTA Trial. J. Thorac. Oncol. 2020, 15, 404-415. [CrossRef] [PubMed]

260. Camidge, D.R.; Kim, H.R.; Ahn, M.J.; Yang, J.C.; Han, J.Y.; Lee, J.S.; Hochmair, M.J.; Li, J.Y.; Gridelli, C.; Delmonte, A.; et al. Brigatinib versus Crizotinib in ALK-Positive Non-Small-Cell Lung Cancer. N. Engl. J. Med. 2018, 379, 2027-2039. [CrossRef]

261. Camidge, D.R.; Kim, H.R.; Ahn, M.J.; Yang, J.C.H.; Han, J.Y.; Hochmair, M.J.; Lee, K.H.; Delmonte, A.; Garcia Campelo, M.R.; Kim, D.W.; et al. Brigatinib Versus Crizotinib in Advanced ALK Inhibitor-Naive ALK-Positive Non-Small Cell Lung Cancer: Second Interim Analysis of the Phase III ALTA-1L Trial. J. Clin. Oncol. 2020, 38, 3592-3603. [CrossRef] [PubMed]

262. Drilon, A.; Siena, S.; Dziadziuszko, R.; Barlesi, F.; Krebs, M.G.; Shaw, A.T.; de Braud, F.; Rolfo, C.; Ahn, M.J.; Wolf, J.; et al. Entrectinib in ROS1 fusion-positive non-small-cell lung cancer: Integrated analysis of three phase 1-2 trials. Lancet Oncol. 2020, 21, 261-270. [CrossRef]

263. Camidge, D.R.; Pabani, A.; Miller, R.M.; Rizvi, N.A.; Bazhenova, L. Management Strategies for Early-Onset Pulmonary Events Associated with Brigatinib. J. Thorac. Oncol. 2019, 14, 1547-1555. [CrossRef]

264. Ng, T.L.; Narasimhan, N.; Gupta, N.; Venkatakrishnan, K.; Kerstein, D.; Camidge, D.R. Early-Onset Pulmonary Events Associated with Brigatinib Use in Advanced NSCLC. J. Thorac. Oncol. 2020, 15, 1190-1199. [CrossRef]

265. Shaw, A.T.; Friboulet, L.; Leshchiner, I.; Gainor, J.F.; Bergqvist, S.; Brooun, A.; Burke, B.J.; Deng, Y.L.; Liu, W.; Dardaei, L.; et al. Resensitization to Crizotinib by the Lorlatinib ALK Resistance Mutation L1198F. N. Engl. J. Med. 2016, 374, 54-61. [CrossRef]

266. Sakamoto, M.R.; Honce, J.M.; Lindquist, D.L.; Camidge, D.R. Lorlatinib Salvages CNS Relapse in an ALK-Positive Non-Small-Cell Lung Cancer Patient Previously Treated with Crizotinib and High-Dose Brigatinib. Clin. Lung Cancer 2019, 20, e133-e136. [CrossRef]

267. Shaw, A.T.; Solomon, B.J.; Besse, B.; Bauer, T.M.; Lin, C.C.; Soo, R.A.; Riely, G.J.; Ou, S.I.; Clancy, J.S.; Li, S.; et al. ALK Resistance Mutations and Efficacy of Lorlatinib in Advanced Anaplastic Lymphoma Kinase-Positive Non-Small-Cell Lung Cancer. J. Clin. Oncol. 2019, 37, 1370-1379. [CrossRef] [PubMed]

268. Shi, R.; Filho, S.N.M.; Li, M.; Fares, A.; Weiss, J.; Pham, N.A.; Ludkovski, O.; Raghavan, V.; Li, Q.; Ravi, D.; et al. BRAF V600E mutation and MET amplification as resistance pathways of the second-generation anaplastic lymphoma kinase (ALK) inhibitor alectinib in lung cancer. Lung Cancer 2020, 146, 78-85. [CrossRef]

269. Coleman, N.; Yousaf, N.; Arkenau, H.T.; Welsh, L.; Popat, S. Lorlatinib Salvages Central Nervous System-Only Relapse on Entrectinib in ROS1-Positive NSCLC. J. Thorac. Oncol. 2020, 15, e142-e144. [CrossRef] [PubMed]

270. Peters, S.; Camidge, D.R.; Shaw, A.T.; Gadgeel, S.; Ahn, J.S.; Kim, D.W.; Ou, S.I.; Perol, M.; Dziadziuszko, R.; Rosell, R.; et al. Alectinib versus Crizotinib in Untreated ALK-Positive Non-Small-Cell Lung Cancer. N. Engl. J. Med. 2017, 377, 829-838. [CrossRef]

271. Yun, M.R.; Kim, D.H.; Kim, S.Y.; Joo, H.S.; Lee, Y.W.; Choi, H.M.; Park, C.W.; Heo, S.G.; Kang, H.N.; Lee, S.S.; et al. Repotrectinib Exhibits Potent Antitumor Activity in Treatment-Naïve and Solvent-Front-Mutant ROS1-Rearranged Non-Small Cell Lung Cancer. Clin. Cancer Res. 2020, 26, 3287-3295. [CrossRef]

272. Schuler, M.; Berardi, R.; Lim, W.T.; de Jonge, M.; Bauer, T.M.; Azaro, A.; Gottfried, M.; Han, J.Y.; Lee, D.H.; Wollner, M.; et al. Molecular correlates of response to capmatinib in advanced non-small-cell lung cancer: Clinical and biomarker results from a phase I trial. Ann. Oncol. 2020, 31, 789-797. [CrossRef] [PubMed] 
273. Drilon, A.; Clark, J.W.; Weiss, J.; Ou, S.I.; Camidge, D.R.; Solomon, B.J.; Otterson, G.A.; Villaruz, L.C.; Riely, G.J.; Heist, R.S.; et al. Antitumor activity of crizotinib in lung cancers harboring a MET exon 14 alteration. Nat. Med. 2020, 26, 47-51. [CrossRef]

274. Paik, P.K.; Drilon, A.; Fan, P.D.; Yu, H.; Rekhtman, N.; Ginsberg, M.S.; Borsu, L.; Schultz, N.; Berger, M.F.; Rudin, C.M.; et al. Response to MET inhibitors in patients with stage IV lung adenocarcinomas harboring MET mutations causing exon 14 skipping. Cancer Discov. 2015, 5, 842-849. [CrossRef] [PubMed]

275. Klempner, S.J.; Borghei, A.; Hakimian, B.; Ali, S.M.; Ou, S.I. Intracranial Activity of Cabozantinib in MET Exon 14-Positive NSCLC with Brain Metastases. J. Thorac. Oncol. 2017, 12, 152-156. [CrossRef]

276. Wolf, J.; Seto, T.; Han, J.Y.; Reguart, N.; Garon, E.B.; Groen, H.J.M.; Tan, D.S.W.; Hida, T.; de Jonge, M.; Orlov, S.V.; et al. Capmatinib in MET Exon 14-Mutated or MET-Amplified Non-Small-Cell Lung Cancer. N. Engl. J. Med. 2020, 383, 944-957. [CrossRef] [PubMed]

277. Paik, P.K.; Felip, E.; Veillon, R.; Sakai, H.; Cortot, A.B.; Garassino, M.C.; Mazieres, J.; Viteri, S.; Senellart, H.; Van Meerbeeck, J.; et al. Tepotinib in Non-Small-Cell Lung Cancer with MET Exon 14 Skipping Mutations. N. Engl. J. Med. 2020, 383, 931-941. [CrossRef]

278. Garon, E.B.; Heist, R.S.; Seto, T.; Han, J.-Y.; Reguart, N.; Groen, H.J.M.; Tan, D.S.W.; Hida, T.; De Jonge, M.J.A.; Orlov, S.V.; et al. Capmatinib in METex14-mutated (mut) advanced non-small cell lung cancer (NSCLC): Results from the phase II GEOMETRY mono-1 study, including efficacy in patients (pts) with brain metastases (BM). In Proceedings of the 111th Annual Meeting of the American Association for Cancer Research 2020 Virtual Meeting, Philadelphia, PA, USA, 27-28 April and 22-24 June 2020. Abstract nr CT2082.

279. Roth, K.G.; Mambetsariev, I.; Salgia, R. Prolonged survival and response to tepotinib in a non-small-cell lung cancer patient with brain metastases harboring MET exon 14 mutation: A research report. Cold Spring Harb. Mol. Case Stud. 2020, 6, a005785. [CrossRef]

280. Takamori, S.; Matsubara, T.; Fujishita, T.; Ito, K.; Toyozawa, R.; Seto, T.; Yamaguchi, M.; Okamoto, T. Dramatic intracranial response to tepotinib in a patient with lung adenocarcinoma harboring MET exon 14 skipping mutation. Thorac Cancer. Thorac. Cancer 2021, 12, 978-980. [CrossRef] [PubMed]

281. Sicklick, J.K.; Kato, S.; Okamura, R.; Schwaederle, M.; Hahn, M.E.; Williams, C.B.; De, P.; Krie, A.; Piccioni, D.E.; Miller, V.A.; et al. Molecular profiling of cancer patients enables personalized combination therapy: The I-PREDICT study. Nat. Med. 2019, 25, 744-750. [CrossRef] 